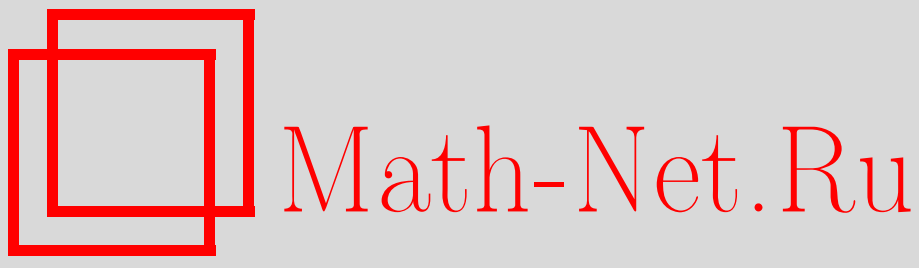

Г. С. Чакветадзе, Топологические и метрические свойства одномерной динамической системы из лазерной физики, Матем. сб., 2002, том 193, номер 8, 101-140

DOI: https://doi.org/10.4213/sm676

Использование Общероссийского математического портала Math-Net.Ru подразумевает, что вы прочитали и согласны с пользовательским соглашением

http://www . mathnet.ru/rus/agreement

Параметры загрузки:

IP: 52.205 .19 .152

26 апреля 2023 г., 14:47:02 
УДК 517.987.5

\author{
Г.С. Чакветадзе
}

\title{
Топологические и метрические свойства одномерной динамической системы из лазерной физики
}

\begin{abstract}
В статье изучаются итерации вещественной рациональной функции $s_{a, b}(x)=$ $b-a x /\left(1+x^{2}\right)$ в зависимости от параметров $a, b \in \mathbb{R}$. Найдены зоны в пространстве параметров, соответствующие динамически тривиальному, а также хаотическому поведению системы. При этом получен аналог теоремы Якобсона для двухпараметрического семейства одномерных отображений, близких к определенному преобразованию с нейтральной неподвижной точкой.

Библиографофия: 12 названий.
\end{abstract}

\section{Введение}

В работе изучается динамическая система на прямой, порожденная рациональной функцией

$$
s_{a, b}(x)=b-\frac{a x}{1+x^{2}},
$$

в зависимости от вешественных параметров $a, b$. Известно, что при $a>0$ итерации $s_{a, b}$ моделируют эволюцию так называемой кавитации Фабри-Перо в лазерах. С прикладной точки зрения наиболее предпочтительными значениями параметров оказываются те, которые соответствуют предельно простому поведению системы (универсальное отталкивающее множество конечно). В этой связи представляет интерес вопрос о сушествовании, расположении и массивности множеств в пространстве параметров, соответствующих поведению хаотическому. Этот вопрос частично исследовался в работе [1] в рамках топологической динамики. В настоящей статье он рассматривается также и с эргодической точки зрения: ищутся множества положительной лебеговой меры в пространстве параметров, соответствующие существованию вероятностной абсолютно непрерывной меры, инвариантной относительно $s_{a, b}$ (далее: к.а.н.и. меры отображения $s_{a, b}$ ). Подобного рода вопросы для обширного класса семейств гладких отображений отрезка широко освещены в литературе, начиная с работы [2]. В представленном исследовании особое внимание уделено случаю, ког да отображения семейства близки к некоторому предельному преобразованию со свойством Мисюревича, обладающему нейтральной неподвижной точкой и, вообще говоря, не имеющему к.а.н.и. меры. Характерно, что несмотря на наличие невырожденных критических точек у рассматриваемых отображений сушествование к.а.н.и. меры в определенном смысле типично даже в этом, если угодно, сильно негиперболическом случае. Похожие явления в иной ситуации недавно обнаружены в работах [3] и [4], где изучались возмущения одномерных отображений в присутствии бифуркаций типа “седло-узел”.

Работа выполнена при частичной поддержке Российского фонда фундаментальных исследований (гранты № 99-01-01104 и № 00-15-96107).

(C) Г. С. Чакветадзе 2002 
Автор выражает глубокую признательность А. М. Степину за постановку задачи и постоянное внимание в процессе ее решения. Кроме того, автор благодарен Д. Ааронсону за дискуссию по поводу преобразований с бесконечной инвариантной мерой и М. Якобсону за полезные замечания, относящиеся к тексту статьи. Часть работы выполнена во время пребывания автора в Институте математики Вроцлавского технологического университета (2000) и в Центре международных математических мероприятий в Люмини, Марсель (2001). Автор благодарит эти учреждения за оказанное гостеприимство и Российский фонд фундаментальных исследований за поддержку упомянутых визитов.

\section{§ 1. Простейшие свойства исследуемых отображений и формулировка результатов}

Приведем простейшие аналитические свойства исследуемой функции:

(1) функция $s_{a, b}$ является вешественно аналитической на всей прямой и имеет две невырожденные критические точки \pm 1 ;

(2) при $a>0$ функция $s_{a, b}$ возрастает на промежутке $(-\infty,-1]$ от $b$ до $b+a / 2$ и на промежутке $[1,+\infty)$ от $b-a / 2$ до $b$, а также убывает на отрезке $[-1,1]$ от $b+a / 2$ до $b-a / 2$, причем $s_{a, b}(0)=b$.

Последнее свойство влечет, что

(3) каждая точка на прямой имеет не более двух прообразов при отображении $s_{a, b}$.

Легко проверяется, что $s_{a, b}$ имеет по крайней мере одну и не более трех неподвижных точек. Для дальнейшего сушественно также, что

(4) $s_{a, b}$ имеет отрицательную производную Шварца, т.е.

$$
\frac{s_{a, b}^{\prime \prime \prime}}{s_{a, b}^{\prime}}-\frac{3}{2}\left(\frac{s_{a, b}^{\prime \prime}}{s_{a, b}^{\prime}}\right)^{2}<0, \quad x \neq \pm 1
$$

(5) отображение $s_{a, b}$ сопряжено с отображением $s_{a,-b}$ с помощью преобразования $x \mapsto-x$;

(6) функция $s_{a, b}$ инвариантна относительно преобразования инверсии, т.е.

$$
s_{a, b}(x)=s_{a, b}\left(\frac{1}{x}\right), \quad x \neq 0 .
$$

Свойство (5) означает, что при исследовании параметрической плоскости для $s_{a, b}$ можно ограничиться значениями $b \geqslant 0$. Свойства (3) и (6) говорят о том, что

(7) если $x_{1}$ и $x_{2}$ являются прообразами одной и той же точки при отображении $s_{a, b}$, то $x_{1}=x_{2}^{-1}$.

Обозначим через $\mathfrak{O}$ пару $(+\infty, 2)$. В $\S 2$ будет доказан следующий результат.

ТЕОРЕМА 1. Вблизи точки $\mathfrak{O}$ существует множсество положительной лебеговой мерь $\mathfrak{S}_{1} \in \mathbb{R}^{2}$ такое, что для любой парь $(a, b) \in \mathfrak{S}_{1}$ отображсение $s_{a, b}$ имеет к.а.н.и. меру. 
ЗАмЕчАнИЕ 1. Сформулированная теорема представляет собой результат теории специального возмущения отображения $V: x \mapsto 2+x+x^{-1}$, имеющего нейтральную неподвижную точку в бесконечности и бесконечную инвариантную меру: ее плотность дается формулой

$$
\rho(x)= \begin{cases}|x|^{-1 / 2}, & x<0, \\ 0, & x \geqslant 0 .\end{cases}
$$

Сужение $\left.V\right|_{(-\infty, 0)}$ консервативно, вполне эргодично, а также поточечно сопряженно-эргодично с последовательностью возвратов

$$
\frac{\sqrt{n}}{2 \sqrt{2} \Gamma\left(\frac{3}{2}\right)^{2}}
$$

(см. [5] по поводу соответствующих определений и результатов теории преобразований, сохраняющих бесконечную меру).

Для того чтобы сформулировать следующий результат, удобнее наряду с семейством $s_{a, b}, a>0, b>0$, рассмотреть семейство $r_{a, d}, a>0, d>-a / 2$, задаваемое формулой

$$
r_{a, d}(x)=d+\frac{a}{2} \frac{(1-x)^{2}}{1+x^{2}} .
$$

Нетрудно видеть, что

$$
r_{a, d}=s_{a, d+a / 2}
$$

Обозначим через $a_{0}=a_{0}(d)$ функцию $d \mapsto \max (0,-2 d)$.

Tеорема 2. Существуют число $d^{\prime} \in(0,1)$, а также дифферениируемые функиии $a_{1}:[-1 / 2,1) \rightarrow \mathbb{R}, a_{2}:(-\infty, 1) \rightarrow \mathbb{R}$ и $a_{3}:\left(d^{\prime}, 1\right) \rightarrow \mathbb{R}$ такие, что

(i) выполнень следующие неравенства: $a_{0}<a_{1}<a_{2}, d \in(-1 / 2,1)$, $a_{0}<a_{2}, d \in(-\infty, 1), u a_{2}<a_{3}, d \in\left(d^{\prime}, 1\right)$

(ii) имеем равенства

$$
\lim _{d \rightarrow 1-0} a_{1}(d)=\lim _{d \rightarrow 1-0} a_{2}(d)=\lim _{d \rightarrow 1-0} a_{3}(d)=\lim _{d \rightarrow-\infty} a_{2}(d)=+\infty
$$

u, кроме того, $a_{1}\left(-2^{-1}\right)=a_{0}\left(-2^{-1}\right)=1$;

(iii) для любой парь $(a, d)$ из множества $\mathfrak{P}_{1}=\left\{(a, d) \in \mathbb{R}^{2}:-1 / 2<d<1\right.$, $\left.a_{0}(d)<a<a_{1}(d)\right\} \cup\left\{(a, d) \in \mathbb{R}^{2}: d \geqslant 1, a>0\right\}$ универсальное отталкивающее множество системы $r_{a, d}$ (см. [6]) либо пусто, либо одноточечно;

(iv) для любой парьи $(a, d)$ из множества $\mathfrak{P}_{2}=\left\{(a, d) \in \mathbb{R}^{2}: d<1\right.$, $\left.a>a_{2}(d)\right\}$ топологическая энтропия системь $r_{a, d}$ положсительна;

(v) вблизи графика $\mathfrak{P}_{3}$ функции а $а$ существует множсество положительной лебеговой мерьи $\mathfrak{S}_{2} \in \mathbb{R}^{2}$ такое, что для любой пары $(a, d) \in \mathfrak{S}_{2}$ отображение $r_{a, d}$ имеет к.а.н.и. меру.

ЗАмечАние 2. Заменив в утверждениях (iii), (iv), (v) множества $\mathfrak{P}_{i}$ их образами под действием преобразования $(a, d) \mapsto(a, d+a / 2)$, получим соответствуюшие результаты для семейства $s_{a, b}, a>0, b>0$.

ЗАмЕчАниЕ 3 . В работе [1] доказан вариант утверждения (iv), в котором вместо множества $\mathfrak{P}_{2}$ фигурируют определенные сегменты прямых, лежащие внутри $\mathfrak{P}_{2}$. 
ЗАмЕчАНИЕ 4. В $\S 3$ найдены выражения для функций $a_{i}$ (см. (3.14)-(3.16)).

ЗАМЕЧАНИЕ 5. Вычисления показывают, что $d^{\prime} \approx 0.317183$.

В дальнейшем символ $\partial$ будет использоваться для обозначения пространственной производной, а символы $\partial_{a}, \partial_{b}$ - для обозначения производных по соответствующим параметрам. Для множества $J \subset \mathbb{R}$ через $\bar{J}$ обозначается его замькание и через $|J|$ - его линейная мера Лебега (в случае, если $J$ измеримо).

\section{§ 2. Доказательство теоремы 1}

В этом параграфе будет доказана теорема 1 для отображения $s_{a,-b}$, что не меняет сути дела согласно свойству (5) функции $s_{a, b}$. Центральное место в построении множества $\mathfrak{S}_{1}$ занимает доказательство следующего результата.

Теорема 3. Существует $c_{0} \gg 1$ такое, что для любого $c \geqslant c_{0}$ существует множество $\mathfrak{G}_{c} \in \mathbb{R}$ положительной лебеговой меры такое, что для любого $b \in \mathfrak{G}_{c}$ отображсене $s_{c(2-b)^{-3},-b}$ имеет к.а.н.и. меру. При этом для любого с точка $b=2$ является левосторонней точкой плотности множества $\mathfrak{G}_{c}$.

Зафиксируем символ $S$ для обозначения отображения $s_{c(2-b)^{-3},-b}^{2} \mid[0,+\infty)$. Отметим, что большое число $c$ в сформулированной теореме играет роль, в некотором смысле аналогичную роли малого параметра в семействе отображений Эно (параметр $b$ в работе [7]). Ключевые оценки доказательства появляются уже при исследовании однопараметрического семейства отображений, получающихся из $S$ при $c=\infty$, каждое из которых имеет нейтральную неподвижную точку в бесконечности. Доказательство в общем случае может быть получено путем выбора такого числа $c_{0}$, что эти оценки выживают при $c \geqslant c_{0}$.

2.1. Метод индуцирования. Пусть $F: \Lambda \rightarrow I$ есть диффеоморфизм своего интервала определения $\operatorname{dom}(F)=\Lambda$ на образ $I$. Обозначим

$$
\tau(F)=\tau(F, \Lambda)=\sup _{x, y \in \Lambda} \frac{\left|F^{\prime}(x)\right|}{\left|F^{\prime}(y)\right|}, \quad \mu(F)=\mu(F, \Lambda)=\sup _{x \in \Lambda} \frac{\left|F^{\prime \prime}(x)\right|}{F^{\prime}(x)^{2}} .
$$

Будем назьвать диффеоморфизм $F$ продолжсаемым до диффеоморфизма на интервал $\widehat{I} \supset I$, если сушествуют интервал $\widehat{\Lambda} \supset \Lambda$ и диффеоморфизм $\widehat{F}: \widehat{\Lambda} \rightarrow \widehat{I}$, имеющий отрицательную производную Шварца и совпадающий с $F$ на $\Lambda$. Пусть при этом $\widehat{I}=L \cup \bar{I} \cup R, L, R \neq \varnothing$. Тогда согласно принципу Кёбе (см. [8]) для любого $\nu>0$ сушествуют числа $\tau=\tau(\nu), \chi=\chi(\nu)$, не зависящие от $F$, такие, что

1) если $\nu<\min \left(\frac{|L|}{|I|}, \frac{|R|}{|I|}\right)$, то $\tau(F, \Lambda) \leqslant \tau$;

2) если при этом интервал $I$ содержит середину интервала $\widehat{I}$, то

$$
\tau(F, \Lambda) \leqslant \exp \left(\chi \frac{|I|}{|\widehat{I}|}\right)
$$

ЗАмечАнИЕ 6 . Можно показать, что в этих условиях сушествует число $\mu<\infty$, зависяшее только от длин $|L|,|R|,|I|$, такое, что $\mu(F, \Lambda) \leqslant \mu$.

Пусть $I$ - интервал, $I_{n}, n \in \mathbb{N}$, - семейство дизъюнктных интервалов, и пусть $G: \bigcup I_{n} \rightarrow I$ - отображение. Для любого $n$ ограничение $\left.G\right|_{I_{n}}$ назьвается ветвъю отображения $G$. В ходе изложения будут возникать ветви двух типов: монотонные (т.е. такие, которые являются диффеоморфизмами на $I$ ) и параболические 
(т.е. такие, каждая из которых представляет собой дифференцируемое унимодальное отображение с невырожденной критической точкой, переводяшее границу своего интервала определения в границу $I)$.

Известно (см., к примеру, [8]), что для доказательства теоремы 3 достаточно для любого $c \gg 1$ построить множество $\mathfrak{G}_{c} \in \mathbb{R}$ такое, что для любого $b \in \mathfrak{G}_{c}$ найдутся интервал $I$, семейство $I_{n}, n \in \mathbb{N}$, дизъюнктных подынтервалов $I$ и отображение $G: \bigcup I_{n} \rightarrow I$, удовлетворяющие следующим свойствам:

(a) $\left|I \backslash \bigcup I_{n}\right|=0$;

(б) все ветви $\left.G\right|_{I_{n}}$ продолжаемы до диффеоморфизмов $\widehat{G}_{n}$ на некоторый фиксированньй интервал $\widehat{I} \supset I$ с тем условием, что множество $\widehat{I} \backslash I$ имеет две нетривиальные компоненты связности и для любого $n \operatorname{dom}\left(\widehat{G}_{n}\right) \subset \widehat{I}$;

(в) для любого $n$ существует $k_{n} \in \mathbb{N}$ такое, что $\left.G\right|_{I_{n}}=S^{k_{n}}$;

(г) числовой ряд $\sum k_{n}\left|I_{n}\right|$ сходится.

Обшая схема построения отображения $G$ заимствована автором из работ [2], [9], однако определенные технические детали отличаются. В частности, предлагаемая конструкция существенно опирается на свойство (4) функции $s_{a, b}$. Она состоит из трех компонент: вычислительной части, предварительной конструкции и основной индуктивной конструкции.

2.2. Вычислительная часть. Заметим, что при $b>1$ ограничение отображения $s_{a,-b}^{2}$ на промежуток $[0,+\infty)$ унимодально, причем $s_{a,-b}^{2}$ убьвает на отрезке $[0,1]$ и возрастает на промежутке $[1,+\infty), s_{a,-b}^{2}(0)=s_{a,-b}^{2}(+\infty)=s_{a,-b}(-b)$. Зафиксируем $\varepsilon, 0<\varepsilon \ll 10^{-1}$, и обозначим через $h$ число $c^{-1}$.

Лемма 1. Существуют числа $c_{1}=c_{1}(\varepsilon), b_{1}=b_{1}(\varepsilon)$ такие, что при $c \geqslant c_{1}$, $b_{1} \leqslant b \leqslant 2$ выполнено неравенство $(1-\varepsilon)(2-b) \leqslant S(1) \leqslant 2-b$.

ДокАЗАтЕльство. Утверждение леммы вытекает из того, что

$$
S(1)=(2-b)\left(1+h(2-b)^{3} \Phi(c, b)\right),
$$

причем существует $C>0$ такое, что $C \leqslant \Phi(c, b) \leqslant 0$ в некоторой окрестности точки $(+\infty, 2)$.

Таким образом, промежуток $[0,+\infty)$ инвариантен относительно $S$ и $S(1)<1$ при надлежащем выборе $c$ и $b$. При этом существует неподвижная точка $p \in(0,1)$ отображения $S$. Положим $I=\left(p, p^{-1}\right)$. Заметим, что в силу свойства (6) функции $s_{a, b}$ точка $p^{-1}$ представляет собой прообраз $p$ под действием $S$.

Лемма 2. Существуют числа $c_{2}=c_{2}(\varepsilon), b_{2}=b_{2}(\varepsilon)$ такие, что при $c \geqslant c_{2}$, $b_{2} \leqslant b \leqslant 2$ выполнены неравенства

$$
\left|p-\frac{1}{2}\right| \leqslant \varepsilon, \quad\left|\frac{1}{p}-2\right| \leqslant \varepsilon, \quad|\partial S(p)+3| \leqslant \varepsilon .
$$

ДоКАЗАТЕЛЬСтво. Лемма следует из того, что для любого $x \neq 0$

$$
\lim _{(a, b) \rightarrow \mathfrak{O}} s_{a,-b}^{2}(x)=s_{\infty,-2}^{2}(x)=x+\frac{1}{x}-2,
$$

причем сходимость равномерна на компактных множествах, не содержащих 0. Легко видеть, что отображение $s_{\infty,-2}^{2}$ имеет неподвижную точку $2^{-1}$ с мультипликатором -3 .

Следующая лемма описывает асимптотику определенных прообразов в окрестности инвариантного интервала $\left(S(1), S^{2}(1)\right)$. 
Лемма 3. Существуют $c_{3}=c_{3}(\varepsilon), b_{3}=b_{3}(\varepsilon)$ такие, что для любого $c \geqslant c_{3}$ определена функиия $N=N_{c}:\left[b_{3}, 2\right) \rightarrow \mathbb{N}$ со следующими свойствами:

(i) для любого $c \quad \lim _{b \rightarrow 2-0} N_{c}(b)=\infty$;

(ii) существует множество $\left\{z_{0}, z_{1}, \ldots, z_{N}\right\}$ такое, что $p^{-1}=z_{0}<z_{1}<$ $\cdots<z_{N}, S\left(z_{n}\right)=z_{n-1}, n=1, \ldots, N$

(iii) существуют множество $\left\{y_{0}, y_{1}, \ldots, y_{N}\right\}$ и число $C_{1}>0$ такие, что $p=y_{0}>y_{1}>\cdots>y_{N}>0, S\left(y_{n}\right)=z_{n-1}, n=1, \ldots, N$, и выполнень оченки

$$
\frac{1}{5 n} \leqslant y_{n} \leqslant \frac{1}{n}, \quad \frac{C_{1}^{-1}}{n^{2}} \leqslant\left|\Lambda_{n}\right| \leqslant \frac{C_{1}}{n^{2}}, \quad n=1, \ldots, N,
$$

əде $\Lambda_{n}=\left(y_{n}, y_{n-1}\right)$;

(iv) nyсmь $M=M_{c}(b)=\max \left\{n \leqslant N: \Lambda_{n} \subset S(I)\right\}$, mогдa

$$
M<\frac{14}{15} N, \quad \lim _{b \rightarrow 2-0} M_{c}(b)=\infty .
$$

ДоказАТельство. (i), (ii) Рассмотрим последовательность, быть может и бесконечную, точек $z_{n}, n=1, \ldots$, таких, что $p^{-1}=z_{0}<z_{1}<\cdots, S\left(z_{n}\right)=z_{n-1}$, $n=1, \ldots$. Пусть

$$
N=\max \left\{n \in \mathbb{N}: \Omega_{n} \text { сушествует и } \Omega_{n} \subset\left(p^{-1}, 15(2-b)^{-1}\right)\right\},
$$

где $\Omega_{n}=\left(z_{n-1}, z_{n}\right)$. Чтобы доказать корректность определения числа $N$ и свойство (i), достаточно установить, что существуют $c_{3}=c_{3}(\varepsilon), b_{3}=b_{3}(\varepsilon)$ такие, что при $c \geqslant c_{3}, b_{3} \leqslant b<2$ для любого $x \in\left[p^{-1}, 16(2-b)^{-1}\right]$ выполнено неравенство

$$
x-2 \leqslant S(x) \leqslant x-1 .
$$

Действительно, в этих условиях для любого $x \in\left(p^{-1}, 15(2-b)^{-1}\right)$ определен единственный прообраз $x^{\prime}$ точки $x$ такой, что $x^{\prime}>x$, причем $x^{\prime} \geqslant x+1$, откуда $N$ конечно. Кроме того, для любого $n$ такого, что $z_{n}$ определено и принадлежит $\left[p^{-1}, 16(2-b)^{-1}\right]$, имеем оценку

$$
1 \leqslant z_{n}-z_{n-1} \leqslant 2
$$

Используя ее последовательно и учитывая лемму 2 , получаем

$$
n+2-\varepsilon \leqslant z_{n} \leqslant 2 n+2+\varepsilon,
$$

откуда выводится неравенство

$$
\frac{15}{2(2-b)}-2-\frac{\varepsilon}{2} \leqslant N \leqslant \frac{15}{2-b}+2-\varepsilon .
$$

Докажем неравенство (2.1). Так как

$$
\begin{aligned}
x-S(x)= & x\left(1-\frac{1+h(2-b)^{3} b\left(x+x^{-1}\right)}{\left(1+h(2-b)^{3} b\left(x+x^{-1}\right)\right)^{2}+h^{2}(2-b)^{6}\left(x+x^{-1}\right)^{2}}\right) \\
& +b-\frac{1}{x} \frac{1+h(2-b)^{3} b\left(x+x^{-1}\right)}{\left(1+h(2-b)^{3} b\left(x+x^{-1}\right)\right)^{2}+h^{2}(2-b)^{6}\left(x+x^{-1}\right)^{2}} \\
= & \mathscr{A}_{1}(c, b, x)+b-\mathscr{A}_{2}(c, b, x),
\end{aligned}
$$


то достаточно показать, что сушествуют $c_{3}=c_{3}(\varepsilon), b_{3}=b_{3}(\varepsilon)$ такие, что при $c \geqslant c_{3}, b_{3} \leqslant b<2$ для любого $x \in\left[p^{-1}, 16(2-b)^{-1}\right]$

$$
0 \leqslant \mathscr{A}_{1} \leqslant 2-b, \quad 0 \leqslant \mathscr{A}_{2} \leqslant b-1
$$

В силу того что $x \geqslant 2-\varepsilon$ и $0<x+x^{-1}<32(2-b)^{-1}$ при $x \in\left[p^{-1}, 16(2-b)^{-1}\right]$, имеем

$$
\begin{aligned}
0<\mathscr{A}_{1} \leqslant & \frac{16}{2-b} \\
\times & \frac{h(2-b)^{3} b \cdot 32(2-b)^{-1}+h^{2}(2-b)^{6}\left(b^{2}+1\right) 32^{2}(2-b)^{-2}}{1-2 h(2-b)^{3} b \cdot 32(2-b)^{-1}-h^{2}(2-b)^{6}\left(b^{2}+1\right) 32^{2}(2-b)^{-2}} \leqslant 2-b \\
& 0<\mathscr{A}_{2} \leqslant \frac{1}{2-\varepsilon}\left(1+h(2-b)^{3} b \cdot 32(2-b)^{-1}\right) \leqslant \frac{3}{4} \leqslant b-1
\end{aligned}
$$

при достаточно малом $\max (h, 2-b)$.

(iii) В силу свойства (6) функции $s_{a, b}$ точки вида $y_{n}=z_{n}^{-1}, n=1, \ldots, N$, удовлетворяют требованиям п. (iii). Учитывая это, не составляет труда вывести оценки п. (iii) из неравенств (2.2) и (2.3). Заметим, что прообразы точек под действием $S$, лежащие на интервале $(0,1)$, определены однозначно, ибо $S$ убывает на $(0,1)$.

(iv) В силу определения числа $M$ выполнено неравенство $S(1) \leqslant y_{M}$. Из леммы 1 получаем, что $(1-\varepsilon)(2-b) \leqslant y_{M}$. Используя оценку на $y_{n}$, отсюда выводим, что $(1-\varepsilon)(2-b) \leqslant M^{-1}$. Принимая во внимание оценку $(2.4)$, из последнего неравенства получаем

$$
M \leqslant(1-\varepsilon)^{-1}(2-b)^{-1} \leqslant 2 \cdot \frac{2}{15}\left(N+2+\frac{\varepsilon}{2}\right) \leqslant \frac{2}{15} N \cdot 7
$$

$M \ll N$. Поэтому число $y_{M+1}$ определено, $y_{M+1}<S(1)$ и аналогично предыдущему получаем, что $M \rightarrow \infty$ равномерно по $c$ при $b \rightarrow 2-0$, что и требовалось доказать.

Заметим, что должны выполняться условия $c_{3} \geqslant \max \left(c_{1}, c_{2}\right), b_{3} \geqslant \max \left(b_{1}, b_{2}\right)$, так как в доказательстве использовались леммы 1 и 2.

Динамическое определение чисел $p^{-1}, y_{n}, z_{n}, n=1, \ldots, N$, и монотонность отображения $S$ на интервалах $(0,1)$ и $(1,+\infty)$ говорят о том, что для любого $n=1, \ldots, N S^{n}$ диффеоморфно отображает интервалы $\Lambda_{n}$ и $\Omega_{n}$ на $I$, причем всюду на $\Lambda_{n}$ и $\Omega_{n} n$ есть момент первого попадания в $I$. Определим отображение $H: \bigcup_{n=1}^{N} \Lambda_{n} \rightarrow I$ по формуле $\left.H\right|_{\Lambda_{n}}=H_{n}=\left.S^{n}\right|_{\Lambda_{n}}, n=1, \ldots, N$, и положим $T=H \circ S$ на множестве $\bigcup_{n=1}^{M+1} \Delta_{n}$, где для любого $n \Delta_{n}=S^{-1}\left(\Lambda_{n}\right)$. Из сказанного вытекает, что $T$ представляет собой отображение первого возврашения на $I$. Заметим, что $T$, вообше говоря, не удовлетворяет всем требованиям, предъявляемњм к отображению $G$, так как, в частности, может обладать центральной параболической ветвью $T_{M+1}=H_{M+1} \circ S$. Подобные ветви будут впоследствии устраняться путем взятия определенных композиций. При этом окажутся важными некоторые свойства диффеоморфизмов $H_{n}$, которые будут суммированы в лемме 5 . Подготовкой к ее доказательству служит формулируемая ниже лемма 4. 
ЗАмечАниЕ 7. Для отображения $S$ при $b=2$ (см. доказательство леммы 2) все сказанное остается в силе за тем исключением, что в лемме 3 числа $N$ и $M$ не определены, соответствующие множества прообразов бесконечны и отображение $T$ обладает бесконечньм числом монотонных ветвей. Полиномиальная асимптотика прообразов $y_{n}$ в этом случае известна и вычислялась, к примеру, в [10] и [11]. Кроме того, $T$ удовлетворяет требованиям (а)-(в), предъявляемым к отображению $G$ (в качестве $\widehat{I}$ берется интервал $(0,+\infty))$.

В дальнейшем для любых целых $i \geqslant 0, j \geqslant 0$ символом $\mathrm{O}\left(h^{i}(2-b)^{j}\right)$ обозначается любая числовая функция $\Phi$ переменных $c, b, x$ со следующим свойством: существует число $C>0$ такое, что для любых $c \geqslant c_{3}, b_{3} \leqslant b \leqslant 2, x \in\left[y_{N}, z_{N}\right]$ вьполнено неравенство $|\Phi(c, b, x)| \leqslant C h^{i}(2-b)^{j}$.

ЛЕмма 4. При $c \geqslant c_{3}, b_{3} \leqslant b \leqslant 2$ выполнены следующие утвержсдения:

(i) $\partial_{b} S=-1+\mathrm{O}(h), \partial_{b} \partial S \cdot(\partial S)^{-1}=\mathrm{O}(h(2-b))$;

(ii) существует $C_{2}=C_{2}\left(c_{3}, b_{3}\right)>0$ такое, что для любих $n \in\{1, \ldots, N\}$, $y \in \Lambda_{n} u z \in \Omega_{n}$ имеем

$$
\left|\frac{\partial^{2} S(y)}{\partial S(y)^{2}}\right| \leqslant \frac{C_{2}}{n}+\mathrm{O}\left(h(2-b)^{3}\right), \quad\left|\frac{\partial^{2} S(z)}{\partial S(z)^{2}}\right| \leqslant \frac{C_{2}}{n^{3}}+\mathrm{O}\left(h(2-b)^{3}\right) .
$$

ДокАЗАТЕльство. (i) После некоторых простых преобразований запишем

$$
\begin{aligned}
\partial_{b} S= & -1+h\left(x+\frac{1}{x}\right)^{2}(2-b)^{2}\left(\frac{2-4 b}{\mathscr{A}_{3}}-\frac{\left(1+h(2-b)^{3} b\left(x+x^{-1}\right)\right) \mathscr{A}_{4}}{\mathscr{A}_{3}^{2}}\right) \\
\partial S= & \left(1-\frac{1}{x^{2}}\right)\left(\frac{1+2 h(2-b)^{3} b\left(x+x^{-1}\right)}{\mathscr{A}_{3}}\right. \\
& \left.-\frac{\left(1+h(2-b)^{3} b\left(x+x^{-1}\right)\right) \mathscr{A}_{5}}{\mathscr{A}_{3}^{2}}\right), \\
\partial \partial_{b} S= & \left(1-\frac{1}{x^{2}}\right)\left[2 h(2-b)^{2}\left(x+\frac{1}{x}\right)\left(\frac{2-4 b}{\mathscr{A}_{3}}-\frac{\left(1+h(2-b)^{3} b\left(x+x^{-1}\right)\right) \mathscr{A}_{4}}{\mathscr{A}_{3}^{2}}\right)\right. \\
& +h(2-b)^{2}\left(x+\frac{1}{x}\right)^{2}\left(-\frac{(2-4 b) \mathscr{A}_{5}}{\mathscr{A}_{3}^{2}}-\frac{h(2-b)^{3} b \mathscr{A}_{4}}{\mathscr{A}_{3}^{2}}\right. \\
& \left.\left.-\frac{\left(1+h(2-b)^{3} b\left(x+x^{-1}\right)\right) \mathscr{A}_{6}}{\mathscr{A}_{3}^{2}}+2 \frac{\left(1+h(2-b)^{3} b\left(x+x^{-1}\right)\right) \mathscr{A}_{4} \mathscr{A}_{5}}{\mathscr{A}_{3}^{3}}\right)\right]
\end{aligned}
$$

где

$$
\begin{aligned}
& \mathscr{A}_{3}=\left(1+h(2-b)^{3} b\left(x+x^{-1}\right)\right)^{2}+h^{2}(2-b)^{6}\left(x+x^{-1}\right)^{2}, \\
& \mathscr{A}_{4}=2\left(1+h(2-b)^{3} b\left(x+x^{-1}\right)\right)(2-4 b)-6 h(2-b)^{3}\left(x+x^{-1}\right), \\
& \mathscr{A}_{5}=2\left(1+h(2-b)^{3} b\left(x+x^{-1}\right)\right) h(2-b)^{3} b+2 h^{2}(2-b)^{6}\left(x+x^{-1}\right), \\
& \mathscr{A}_{6}=2 h(2-b)^{3} b(2-4 b)-6 h(2-b)^{3} .
\end{aligned}
$$

Так как $2 \leqslant x+x^{-1} \leqslant 15(2-b)^{-1}+15^{-1}(2-b)$ при $x \in\left[y_{N}, z_{N}\right] \subset\left[15^{-1}(2-b)\right.$, $\left.15(2-b)^{-1}\right]$, то

$$
\mathscr{A}_{3}=1+\mathrm{O}\left(h(2-b)^{2}\right), \quad \mathscr{A}_{4}=\mathrm{O}(1), \quad \mathscr{A}_{5}=\mathrm{O}\left(h(2-b)^{3}\right), \quad \mathscr{A}_{6}=\mathrm{O}\left(h(2-b)^{3}\right) .
$$


Отсюда $\partial_{b} S=-1+\mathrm{O}(h)$. Кроме того,

$$
\frac{\partial_{b} \partial S}{\partial S}=\frac{\left(1-x^{-2}\right)\left(\mathrm{O}(h(2-b))+\mathrm{O}\left(h(2-b)^{3}\right)\right)}{\left(1-x^{-2}\right)\left(1+\mathrm{O}\left(h(2-b)^{2}\right)\right)}=\mathrm{O}(h(2-b)),
$$

что и требовалось доказать. Заметим, что как $\mathscr{A}_{3}$, так и вторая скобка в выражении (2.5) должны быть не меньше $2^{-1}$, что может быть достигнуто за счет подходящего изменения чисел $c_{3}$ и $b_{3}$.

(ii) Вычислив $\partial^{2} S$ и используя выражение (2.5), запишем

$$
\begin{aligned}
\frac{\partial^{2} S(x)}{\partial S(x)^{2}}= & \frac{2 x^{-3}}{\left(1-x^{-2}\right)^{2}}\left(\frac{1+2 h(2-b)^{3} b\left(x+x^{-1}\right)}{\mathscr{A}_{3}}\right. \\
& \left.-\frac{\left(1+h(2-b)^{3} b\left(x+x^{-1}\right)\right) \mathscr{A}_{5}}{\mathscr{A}_{3}^{2}}\right)^{-1}+\mathrm{O}\left(h(2-b)^{3}\right) \\
\leqslant & \frac{2}{z_{n-1}^{3}} \frac{1}{\left(1-(2-\varepsilon)^{-2}\right)^{2}}\left(1+\mathrm{O}\left(h(2-b)^{2}\right)\right)+\mathrm{O}\left(h(2-b)^{3}\right) \\
\leqslant & \frac{2}{(n-1+2-\varepsilon)^{3}}\left(\frac{9}{5}\right)^{2}\left(1+\mathrm{O}\left(h(2-b)^{2}\right)\right)+\mathrm{O}\left(h(2-b)^{3}\right)
\end{aligned}
$$

если $x \in \Omega_{n}, n=1, \ldots, N$ (при этом были использованы малость $\varepsilon$ и оценка $(2.3)$ ). Отсюда следует второе из неравенств п. (ii). Первое из них проверяется аналогично с учетом того, что $y_{n} \leqslant n^{-1}$.

ЗАмЕчАнИЕ 8 . Не ограничивая общности, будем считать, что при $c \geqslant c_{3}, b_{3} \leqslant$ $b \leqslant 2$ выполнены оценки $1-\varepsilon<\left|\partial_{b} S\right|<1+\varepsilon$ и $\left|\partial_{b} \partial S\right||\partial S|^{-1}<\varepsilon$ на промежутке $\left[y_{N}, z_{N}\right]$.

Заметим, что все диффеоморфизмы $H_{n}$, так же как и их интервалы определения, зависят от $c$ и от $b$. Следуюший результат описывает некоторые свойства диффеоморфизмов $H_{n}$, относящиеся к их зависимости от $b$.

Лемма 5. Существуют константы $C_{i}>0, i=3,4,5$, такие, что для любых $c \geqslant c_{3}, b_{3} \leqslant b<2, n=1, \ldots, N$ справедливы следующие оценки:

(i) $\inf _{y \in \Lambda_{n}}\left|\partial H_{n}(y)\right| \geqslant C_{3} n^{2}$;

(ii) $\sup _{y \in \Lambda_{n}}\left|\frac{\partial_{b} H_{n}(b, y)}{\partial H_{n}(b, y)}\right| \leqslant C_{4} n^{-1}$;

(iii) $\sup _{x \in I}\left|\frac{\partial_{b} \partial H_{n}^{-1}(b, x)}{\partial H_{n}^{-1}(b, x)}\right| \leqslant C_{5}$.

ДокАЗАТЕльство. (i) Обозначим $p=z_{-1}, \Omega_{0}=\left(z_{-1}, z_{0}\right)=I$. Зафиксируем $n \geqslant 2$ и заметим, что диффеоморфизм $\left.S^{n-1}\right|_{\Lambda_{n}}$ продолжаем до диффеоморфизмана интервал $\left(z_{-1}, z_{2}\right)$. При этом отношения длин интервалов $\Omega_{0}, \Omega_{1}, \Omega_{2}$ оцениваются ненулевыми числами, не зависящими от $c, b$ (см. лемму 2 и неравенство (2.2)). Это влечет существование числа $\tau$ такого, что $\tau\left(\left.S^{n-1}\right|_{\Lambda_{n}}\right) \leqslant \tau$ для любого $n \geqslant 2$. Используя оценку на $\left|\Lambda_{n}\right|$ из п. (iii) леммы 3 , получаем квадратичную оценку снизу для $\left.\partial S^{n-1}\right|_{\Lambda_{n}}$, которая влечет п. (i). Используя аналогичные рассуждения, можно считать, что для любых $n \in\{1, \ldots, N\}, k \in\{1, \ldots, n\}$

$$
\inf _{z \in \Omega_{n}}\left|\partial S^{k}(z)\right| \geqslant C_{3}, \quad \inf _{y \in \Lambda_{n}}\left|\partial S^{k}(y)\right| \geqslant C_{3} n^{2} .
$$


(ii) Для композиции отображений

$$
\varphi(b, y)=\varphi_{n}\left(b, \varphi_{n-1}\left(b, \ldots \varphi_{2}\left(b, \varphi_{1}(b, y)\right) \ldots\right)\right)
$$

располагаем формулой (см. [2])

$$
\frac{\partial_{b} \varphi(b, y)}{\partial \varphi(b, y)}=\sum_{i=1}^{n} \frac{\partial_{b} \varphi_{i}\left(b, \varphi^{i-1}(b, y)\right)}{\partial\left(\varphi^{i}(b, y)\right)}
$$

где $\varphi^{i}(b, y)=\varphi_{i}\left(b, \varphi_{i-1}\left(b, \ldots \varphi_{2}\left(b, \varphi_{1}(b, y)\right) \ldots\right)\right)$ для любого $i$. Используя эту формулу и опуская зависимость от параметра, запишем

$$
\frac{\partial_{b} H_{n}(y)}{\partial H_{n}(y)}=\frac{\partial_{b} S(y)}{\partial S(y)}+\sum_{i=2}^{n} \frac{\partial_{b} S\left(S^{i-1}(y)\right)}{\partial S^{i}(y)}, \quad y \in \Lambda_{n},
$$

откуда с помощью леммы 4 получим

$$
\left|\frac{\partial_{b} H_{n}(y)}{\partial H_{n}(y)}\right| \leqslant(1+\mathrm{O}(h))\left(\frac{1}{C_{3} n^{2}}+(n-1) \frac{1}{C_{3} n^{2}}\right)=(1+\mathrm{O}(h)) \frac{C_{3}^{-1}}{n},
$$

что и требовалось доказать.

(iii) Пусть $x=S(y)$ и $y \neq 1$. Тогда справедливо выражение

$$
\frac{\partial_{b} \partial S^{-1}(x)}{\partial S^{-1}(x)}=\frac{\partial^{2} S(y)}{\partial S(y)^{2}} \partial_{b} S(y)-\frac{\partial_{b} \partial S(y)}{\partial S(y)}
$$

В обозначениях предыдущего пункта проверяется формула

$$
\begin{aligned}
& \frac{\partial_{b} \partial \varphi^{-1}(b, x)}{\partial \varphi^{-1}(b, x)}=\sum_{i=1}^{n}\left(\frac{\partial_{b} \partial \varphi_{i}^{-1}\left(b, \varphi^{i}(b, y)\right)}{\partial \varphi_{i}^{-1}\left(b, \varphi^{i}(b, y)\right)}\right. \\
& \left.\quad-\frac{\partial^{2} \varphi_{i}\left(b, \varphi^{i-1}(b, y)\right)}{\partial \varphi_{i}\left(b, \varphi^{i-1}(b, y)\right)^{2}} \partial_{b}\left(\varphi_{i+1}^{-1} \circ \cdots \circ \varphi_{n}^{-1}(b, x)\right)\right)
\end{aligned}
$$

где $x=\varphi(b, y), \varphi_{i+1}^{-1} \circ \cdots \circ \varphi_{n}^{-1}(b, x)=\varphi_{i+1}^{-1}\left(b, \ldots \varphi_{n}^{-1}(b, x) \ldots\right)$. Поэтому

$$
\begin{aligned}
\frac{\partial_{b} \partial H_{n}^{-1}(x)}{\partial H_{n}^{-1}(x)}= & \sum_{i=1}^{n-1}\left(\frac{\partial_{b} \partial S^{-1}\left(S^{i}(y)\right)}{\partial S^{-1}\left(S^{i}(y)\right)}-\frac{\partial^{2} S\left(S^{i-1}(y)\right)}{\partial S\left(S^{i-1}(y)\right)^{2}} \partial_{b}\left(\left(\left.S^{n-i}\right|_{\Omega_{n-i}}\right)^{-1}\right)(x)\right) \\
& +\frac{\partial_{b} \partial S^{-1}(x)}{\partial S^{-1}(x)}
\end{aligned}
$$

где $x \in I, y \in \Lambda_{n}, x=H_{n}(y)$. Используя формулу (2.7) и лемму 4 , оценим

$$
\begin{gathered}
\left|\partial_{b}\left(\left(S^{k} \mid \Omega_{k}\right)^{-1}\right)(x)\right|=\left|\frac{\partial_{b} S^{k}(z)}{\partial S^{k}(z)}\right| \leqslant \sum_{j=1}^{k} \frac{\left|\partial_{b} S\left(S^{j-1}(z)\right)\right|}{\partial S^{j}(z)} \leqslant(1+\mathrm{O}(h)) C_{3}^{-1} k, \\
k=1, \ldots, n-1,
\end{gathered}
$$


где $x=S^{k}(z), z \in \Omega_{k}$. Обозначив $y_{i}=S^{i}(y) \in \Omega_{n-i}, i=0,1, \ldots, n-1$, а также принимая во внимание лемму 4 и соотношения $(2.8),(2.10),(2.11)$, запишем

$$
\begin{aligned}
& \left|\frac{\partial_{b} \partial H_{n}^{-1}(x)}{\partial H_{n}^{-1}(x)}\right| \leqslant \sum_{i=1}^{n-1}\left(\left|\frac{\partial^{2} S\left(y_{i-1}\right)}{\partial S\left(y_{i-1}\right)^{2}} \partial_{b} S\left(y_{i-1}\right)-\frac{\partial_{b} \partial S\left(y_{i-1}\right)}{\partial S\left(y_{i-1}\right)}\right|\right. \\
& \left.+\left|\frac{\partial^{2} S\left(y_{i-1}\right)}{\partial S\left(y_{i-1}\right)^{2}}\right|(1+\mathrm{O}(h)) C_{3}^{-1}(n-i)\right) \\
& +\left|\frac{\partial^{2} S\left(y_{n-1}\right)}{\partial S\left(y_{n-1}\right)^{2}} \partial_{b} S\left(y_{n-1}\right)-\frac{\partial_{b} \partial S\left(y_{n-1}\right)}{\partial S\left(y_{n-1}\right)}\right| \\
& \leqslant \sup _{x \in\left[y_{N}, z_{N}\right]}\left|\partial_{b} S(x)\right|\left(\left|\frac{\partial^{2} S(y)}{\partial S(y)^{2}}\right|+\sum_{i=2}^{n}\left|\frac{\partial^{2} S\left(y_{i-1}\right)}{\partial S\left(y_{i-1}\right)^{2}}\right|\right) \\
& +(1+\mathrm{O}(h)) C_{3}^{-1}\left(\left|\frac{\partial^{2} S(y)}{\partial S(y)^{2}}\right|(n-1)+\sum_{i=2}^{n-1}\left|\frac{\partial^{2} S\left(y_{i-1}\right)}{\partial S\left(y_{i-1}\right)^{2}}\right|(n-i)\right) \\
& +n \sup _{x \in\left[y_{N}, z_{N}\right]}\left|\frac{\partial_{b} \partial S(x)}{\partial S(x)}\right| \leqslant(1+\mathrm{O}(h))\left(\frac{C_{2}}{n}+\mathrm{O}\left(h(2-b)^{3}\right)\right. \\
& \left.+\sum_{i=2}^{n}\left(\frac{C_{2}}{(n-i+1)^{3}}+\mathrm{O}\left(h(2-b)^{3}\right)\right)\right) \\
& +(1+\mathrm{O}(h)) C_{3}^{-1}\left(\left(\frac{C_{2}}{n}+\mathrm{O}\left(h(2-b)^{3}\right)\right)(n-1)\right. \\
& \left.+\sum_{i=2}^{n-1}\left(\frac{C_{2}}{(n-i+1)^{3}}+\mathrm{O}\left(h(2-b)^{3}\right)\right)(n-i)\right)+n \mathrm{O}(h(2-b)) \\
& \leqslant(1+\mathrm{O}(h))\left(\frac{C_{2}}{n}\left(1+C_{3}^{-1}(n-1)\right)+\mathrm{O}\left(h(2-b)^{3}\right)\left(n+C_{3}^{-1} \sum_{i=1}^{n-1} i\right)\right. \\
& \left.+C_{2} \sum_{i=1}^{\infty} \frac{1}{i^{3}}+C_{3}^{-1} C_{2} \sum_{i=1}^{\infty} \frac{1}{i^{2}}\right)+\operatorname{NO}(h(2-b)) \leqslant \text { const }
\end{aligned}
$$

так как

$$
\sum_{i=1}^{n-1} i \leqslant n^{2}, \quad n \leqslant N \leqslant 15(2-b)^{-1}(1+\mathrm{O}(2-b)) .
$$

Заметим, что константы $C_{4}$ и $C_{5}$ зависят от $c_{3}, b_{3}$. Будем считать, что $C_{4}>1$.

2.3. Предварительная конструкция. На протяжении пा. 2.3-2.6 все рассмотрения проводятся для некоторого фиксированного значения $c \geqslant c_{3}$. На начальном шаге индуктивной конструкции построения отображения $G$ требуется, чтобы большая часть интервала $I$ была занята интервалами определения сильно растягивающих диффеоморфизмов. Так как периферические ветви отображения $T$ не обязаны быть сильно растягивающими, то $T$, вообще говоря, непригодно в качестве начального приближения к отображению $G$ и нуждается в модификации (см. [9]), которая проделывается следующим образом. Напомним, что для любого $n \in\{1, \ldots, M\}$ множество $\Delta_{n}$ состоит из двух компонент связности, которые с этого момента не различаются и имеют одно и то же обозначение $\Delta_{n}$; символ $T_{n}$ будет использоваться для обозначения каждой из ветвей $\left.T\right|_{\Delta_{n}}$. Наряду с этим символ 
$T_{l}\left(T_{r}\right)$ служит для обозначения той ветви $T_{1}$, которая определена в непосредственной близости к точке $p\left(p^{-1}\right)$.

Для заданного $E>0$ назовем диффеоморфизм $F: \Lambda \rightarrow I E$-растягивающим, если $\inf _{x \in \Lambda}\left|F^{\prime}(x)\right| \geqslant E$. Пусть $b=2$ и $E \gg 1$. В силу сказанного в замечании 7 соответствующее отображение $T$ имеет к.а.н.и. меру. Используя свойство (4) функции $s_{a, b}$, отсюда можно вывести существование такого $L=L(E)$, что для любого $x \in \operatorname{dom}\left(T^{L}\right)\left|\left(T^{L}\right)^{\prime}(x)\right| \geqslant 2 E$. Пусть для некоторого набора $\left(n_{1}, \ldots, n_{i}\right) \in \mathbb{N}^{i}$ диффеоморфизм $U=T_{n_{i}} \circ \cdots \circ T_{n_{1}}: \Delta \rightarrow I$ не является $2 E$-растягивающим. Тогда $i<L$ и существует $K=K(E)$ такое, что для любого $k \in\{1, \ldots, i\} n_{k} \leqslant K$, так как ветви $T_{n}$ с большими $n$ сильно растягивают. Здесь в первый раз возникает следуюшее понятие. Композииией в интервал $\Delta$ отображения $T$ назьвается переход к рассмотрению отображения $W=T \circ U: \bigcup U^{-1}\left(\Delta_{n}\right) \rightarrow I$. Сделаем композицию в $\Delta$. При этом вблизи прообраза $u=U^{-1}(1)$ расположены $2 E$-растягивающие ветви отображения $W$, которые не участвуют в дальнейших композициях. В то же время, при наличии некоторой ветви отображения $W$, находящейся в условиях диффеоморфизма $U$, делается композиция (отображения $T$ ) в ее интервал определения и т.д. Из сказанного ясно, что за конечное число шагов $\left(\leqslant K^{L}\right)$ можно построить отображение $\bar{T}$, совпадающее с $T$ вблизи точки 1 , все ветви которого будут $2 E$-растягивающими. При этом получим конечное множество интервалов, в которые на каком-либо шаге делалась композиция, с определенньми на этих интервалах диффеоморфизмами. Каждый интервал из этого множества будем обозначать через $\Delta_{0}$, а соответствуюший диффеоморфизм - через $T_{0}$. Заметим, что любой интервал $\Delta_{0}$ содержит прообраз $u=\left(T_{0}\right)^{-1}(1)$ и геометрические свойства отображения $\bar{T}$ вблизи $u$ и отображения $T$ около точки 1 одинаковы. При малом изменении $b$ производные ветвей отображения $T$ сушественно меняются только вблизи единицы. Стало быть, значительное изменение производных ветвей отображения $\bar{T}$ происходит только около единицы и конечного числа прообразов $u$. Как результат, большая часть интервала $I$ будет занята областями определения $E$-растягивающих ветвей отображения $\bar{T}$.

Описанное построение называется $E$-предварительной конструкиией. Отметим, что однозначно определены отображение $\bar{H}:\left[y_{N}, p\right] \rightarrow I$, интервалы $\Lambda_{0}$, диффеоморфизмы $H_{0}: \Lambda_{0} \rightarrow I$ такие, что

$$
\left.\bar{H}\right|_{\bigcup_{K<n \leqslant N} \Lambda_{n}}=H, \quad \bar{T}=\bar{H} \circ S, \quad \Delta_{0}=S^{-1}\left(\Lambda_{0}\right), \quad T_{0}=H_{0} \circ S
$$

а также точки $v=\left(H_{0}\right)^{-1}(1)$, вблизи которых геометрические свойства $\bar{H}$ аналогичны соответствующим свойствам $T$ около точки 1.

2.4. Основная индуктивная конструкция. Выбор параметров. Пусть $b=2$. Тогда $I=\left(2^{-1}, 2\right)$ и

$$
\partial S(p)=-3
$$

Положим $\widehat{I}=\left(x_{1}, x_{2}\right)$, где $x_{1}$ и $x_{2}$ удовлетворяют соотношениям

$$
0 \ll x_{1}<\frac{1}{2}, \quad 2<x_{2} \ll 3, \quad x_{1}<x_{1}^{\prime}<\frac{1}{2}, \quad 2<x_{1}^{\prime \prime}<x_{2},
$$

а точки $x_{1}^{\prime}, x_{1}^{\prime \prime}$ однозначно определяются условиями

$$
S^{2}\left(x_{1}^{\prime}\right)=S^{2}\left(x_{1}^{\prime \prime}\right)=x_{1}, \quad S\left(x_{1}^{\prime}\right)=S\left(x_{1}^{\prime \prime}\right) \in(0,1) .
$$


Такой выбор возможен вследствие равенства (2.12). Через $I^{\prime}$ обозначим интервал $\left(x_{1}^{\prime}, x_{1}^{\prime \prime}\right), I \subset I^{\prime} \subset \widehat{I}$.

В дальнейшем любой диффеоморфизм $F: J \rightarrow I$, продолжаемњй до диффеоморфизма на $\widehat{I}$, будем называть просто продолжсаемым и использовать символ $\widehat{J}$ для обозначения интервала определения продолжения $\widehat{F}$. При этом диффеоморфизмы $H_{n}, n=1, \ldots, N$, оказьваются продолжаемыми. Не сужая общности, можно дополнительно считать, что

1) для любого $n \geqslant 2$ вьполнено включение $\widehat{\Lambda}_{n} \subset(0, p)$;

2) для любых $m, n$ таких, что $|m-n|>1$, имеем $\widehat{\Lambda}_{n} \cap \widehat{\Lambda}_{m}=\varnothing$.

Пусть $F: J \rightarrow I$ есть продолжаемый диффеоморфизм такой, что $J \subset(0, p)$. Будем называть диффеоморфизм $F$ и интервал $J$ накрываемымми, если $S\left(I^{\prime}\right) \supset \widehat{J}$. Диффеоморфизм $F$ и интервал $J$ называются критическими, если они не являются накрываемьми, $\widehat{J} \subset(0, p)$ и $J \cap S(I) \neq \varnothing$. Если диффеоморфизм $F$ накрываемый, то диффеоморфизм $F \circ S: \Delta \rightarrow I, \Delta=S^{-1}(J)$, является продолжаемым, причем $\widehat{\Delta}=S^{-1}(\widehat{J}) \subset I^{\prime}$. Из сказанного нетрудно видеть, что ветви $H_{n}, n=1, \ldots, M-1$, являются накрьваемьми, тогда как ветвь $H_{M}$, вообше говоря, может быть и критической (ветвь $H_{M+1}$, очевидно, критическая). Кроме того, $S^{-1}\left(\widehat{\Lambda}_{n}\right) \subset I$ для любого $n$ такого, что $M-1 \geqslant n \geqslant 2$. Поэтому отображение $T$ может иметь только три непродолжаемые ветви: центральную параболическую и соседние с ней, причем $\widehat{\Delta}_{1} \subset I^{\prime}$ и для любого $n \in\{2, \ldots, M-1\}$ имеем включение $\widehat{\Delta}_{n} \subset I$.

Пусть $2^{-1} \tau\left(2^{-1} \tau_{1}\right)$ представляет собой константу из принщипа Кёбе, подходящую для пары интервалов $\left(2^{-1}, 2\right)$ и $\widehat{I}$ (соответственно $I^{\prime}$ и $\left.\widehat{I}\right)$. Тогда при рассматриваемом малом возмушении $b$ для любого продолжаемого диффеоморфизма $F$ имеем $\tau\left(F, F^{-1}(I)\right) \leqslant \tau$ и $\tau\left(\widehat{F}, \widehat{F}^{-1}\left(I^{\prime}\right)\right) \leqslant \tau_{1}$.

Зафиксируем числа $\varkappa, \lambda$ такие, что $0<\varkappa<10^{-1} \lambda<100^{-1}$, и сделаем $E$-предварительную конструкцию, где $E=\tau \varkappa^{-1}$. В обозначениях предыдущего пункта отображение $\bar{T}(\bar{H})$ имеет конечное число непродолжаемых ветвей вблизи прообразов $u(v)$. Обозначим через $\bar{T}_{l}, \bar{T}_{r}$ монотонные ветви отображения $\bar{T}$, определенные в непосредственной близости от точек $p$ и $p^{-1}$ соответственно, $\Delta_{l}=$ $\operatorname{dom}\left(\bar{T}_{l}\right)=\left(p, x^{\prime}\right), \Delta_{r}=\operatorname{dom}\left(\bar{T}_{r}\right)=\left(x^{\prime \prime}, p^{-1}\right), x^{\prime}, x^{\prime \prime} \in I$. Тогда $\widehat{\Delta}_{l} \subset I^{\prime}, \widehat{\Delta}_{r} \subset I^{\prime}$ и если $\left.\bar{T}\right|_{\Delta^{\prime}}$ является какой-либо из остальных продолжаемых ветвей отображения $\bar{T}$, то $\widehat{\Delta}^{\prime} \subset I$. Это нетрудно установить по индукции, учитывая, что аналогичным свойством обладают ветви отображения $T$ и на каждом шаге предварительной конструкции делается композиция $T$ в интервал определения продолжаемого диффеоморфизма. Отметим, что $\bar{T}_{l}=T_{l} \circ \cdots \circ T_{l} \circ T_{l}, \bar{T}_{r}=T_{l} \circ \cdots \circ T_{l} \circ T_{r}$. Поэтому, опираясь на равенство $(2.12)$ и уменьшая в случае необходимости $\varkappa$, можно считать, что $\max \left(\left|\widehat{\Delta}_{l} \backslash I\right|,\left|\widehat{\Delta}_{r} \backslash I\right|\right)<\left(10 \tau_{1}\right)^{-1}|I|$.

С учетом сказанного в п. 2.3 все ветви отображения $\bar{T}$, за исключением определенных на ничтожно малых интервалах вблизи точки $x=1$ и ее прообразов $u$, являются $E$-растягивающими. Поэтому сделанный выбор $E$ означает, что при композиции отображения $\bar{T}$ в интервал определения $\Lambda$ продолжаемого диффеоморфизма $F$ дли́ны возникающих интервалов меньше $|\Lambda|$ по крайней мере в $\varkappa^{-1}$ раз. Кроме того, имеем $\max \left(\left|\widehat{F}^{-1}\left(\widehat{\Delta}_{l}\right) \backslash \Lambda\right|,\left|\widehat{F}^{-1}\left(\widehat{\Delta}_{r}\right) \backslash \Lambda\right|\right)<10^{-1}|\Lambda|$ в силу принципа Кёбе и определения числа $\tau_{1}$.

Зафиксируем натуральные числа $P, P_{1}$ такие, что

$$
C_{1} B_{1} B_{2} \sqrt{\lambda}^{P}<\frac{1}{2}
$$


и $P_{1} \gg P$, где $B_{1}=C_{4}+1+\varepsilon, B_{2}=\tau(2|I|(1-\varepsilon))^{-1}$, константы $C_{1}$ и $C_{4}$ введены в леммах п. 2.2.

Основные составляюшие индуктивной конструкции. В доказательстве теоремы 3 для любого достаточно малого числа $\alpha>0$ строится множество $\mathfrak{G}(\alpha)=$ $\mathfrak{G}_{c}(\alpha)$ такое, что для любого $b \in \mathfrak{G}(\alpha)$ существуют последовательности множеств $\mathfrak{k}_{n}$ интервалов из $(S(1), p)$ и отображений $F_{n}: \bigcup_{\Lambda \in \mathfrak{k}_{n}} \Lambda \rightarrow I, n \in \mathbb{N} \cup\{0\}$, со следующими свойствами:

(i) для любого $n$ множество $\mathfrak{k}_{n}$ конечно;

(ii) для произвольных $m, n \in \mathbb{N} \cup\{0\}, m<n$, выполнено включение $\mathfrak{k}_{m} \subset \mathfrak{k}_{n}$, причем для любого $\left.\Lambda \in \mathfrak{k}_{m} \quad F_{m}\right|_{\Lambda}=\left.F_{n}\right|_{\Lambda}$;

(iii) для каждого $n$ любой интервал из множества $\mathfrak{k}_{n}$ является накрываемым;

(iv) для любого $n$ и для каждого $\Lambda \in \mathfrak{k}_{n}$ найдется $k=k_{\Lambda}$ такое, что $\left.F_{n}\right|_{\Lambda}=S^{k}$.

Пусть для любого $n$ множество $\mathfrak{l}_{n}$ интервалов из $I$ задается равенством $\mathfrak{l}_{n}=\{\Delta$ : $\left.S(\Delta) \in \mathfrak{k}_{n}\right\}$. Определим отображения $G_{n}: \bigcup_{\Delta \in \mathfrak{l}_{n}} \Delta \rightarrow I$ по формуле $G_{n}=F_{n} \circ S$. Тогда имеем:

(v) числовая последовательность $\left|I \backslash \bigcup_{\Delta \in \mathfrak{I}_{n}} \Delta\right|$ стремится к нулю при $n \rightarrow \infty$ экспоненциально равномерно по $c, b$ вблизи точки $\mathfrak{O}$;

(vi) любая ветвь отображения $G_{n}$ продолжаема и интервал определения продолжения лежит внутри $I^{\prime}$.

Нетрудно видеть, что свойство (vi) является непосредственным следствием свойства (iii) и что наличия последовательности $G_{n}, n \in \mathbb{N} \cup\{0\}$, достаточно для сушествования отображения $G$ со свойствами (а)-(в).

Множество $\mathfrak{G}(\alpha)$ представляется в виде $\bigcap_{n \in \mathbb{N} \cup\{0\}} \mathfrak{D}_{n}$, где $\mathfrak{D}_{n}, n \in \mathbb{N} \cup\{0\}$, является убываюшей последовательностью множеств со следующими свойствами:

(vii) для любого $n$ множество $\mathfrak{D}_{n}$ является объединением дизъюнктных интервалов $\mathfrak{E}_{n}$, причем каждый отрезок, не содержаший точку $b=2$, пересекается не более чем с конечньм их числом;

(viii) числовая последовательность $\left|\mathfrak{D}_{n} \backslash \mathfrak{D}_{n+1}\right|, n \in \mathbb{N} \cup\{0\}$, убывает по $n$ экспоненциально равномерно по $c$, причем ее первьй член не превосходит const $\sqrt{\alpha}$

(ix) для любых $n \in \mathbb{N} \cup\{0\}, b \in \mathfrak{D}_{n}$ определены множество $\mathfrak{k}_{n}$ и отображение $F_{n}$.

Множество $\mathfrak{D}_{0}$. Примем для удобства изложения следуюшее соглашение. Пусть $\Pi=\Pi\left(y_{n}, \Lambda_{n}, H_{n}\right)$ есть какое-либо условие на число $n \in \mathbb{N}$. Считается, что $n$ удовлетворяет условию П, если ввиду оценок леммы 3 или леммы $5 n$ удовлетворяет этому условию для всех $c$ и $b$ таких, что $c \geqslant c_{3}, b_{3} \leqslant b<2$ и $N_{c}(b)>n$.

Не ограничивая общности, будем считать, что $K>100$, где $K=K(E)$ введено в п. 2.3, и что для любого $y \in\left(S(1), y_{K}\right)$ вьполнены неравенства

$$
\begin{gathered}
\sqrt{y-S(1)}(1-\varepsilon)<|x-1|<\sqrt{y-S(1)}(1+\varepsilon), \\
2 \sqrt{y-S(1)}(1-\varepsilon)<|\partial S(x)|<2 \sqrt{y-S(1)}(1+\varepsilon)
\end{gathered}
$$

при условии, что $S(x)=y$ (см. доказательство леммы 2$)$.

Найдем число $m_{0}>K$ такое, что для любого $n>m_{0}$

$$
\max \left(\sup _{y \in \Lambda_{n}}\left|\frac{\partial_{b} H_{n}(b, y)-\partial_{b} p^{-1}(b)}{\partial H_{n}(b, y)}\right|, \sup _{y \in \Lambda_{n}}\left|\frac{\partial_{b} H_{n}(b, y)-\partial_{b} p(b)}{\partial H_{n}(b, y)}\right|\right)<\frac{1}{10} \text {. }
$$

Зафиксируем число $\alpha<y_{m_{0}} 2^{-1} \lambda^{P+3}$. 
Излагаемая конструкция индуцирования базируется на определенных оценках, в основном собранных в лемме 8 и зависящих от параметров $\alpha$ и $\beta, \beta>0$. В свою очередь, эти оценки возможны благодаря тому, что частное чисел $\beta$ и $\alpha$ достаточно мало. Для диффеоморфизма $F: \Lambda \rightarrow I$, зависящего от параметра $b$, будем обозначать

$$
\mathscr{V}(F)=\mathscr{V}(F, \Lambda)=\sup _{y \in \Lambda}\left|\frac{\partial_{b} F(b, y)}{\partial F(b, y)}\right| .
$$

Выберем числа $A_{1}>0, A_{2}>0, A_{3}>0, A_{4}>0$ такие, что

$$
\max \left(A_{1}+A_{2}, A_{1}+A_{3}+A_{4}\right)<\left(\frac{1+\varepsilon}{C_{4}}+1\right)^{-1},
$$

и зафиксируем число $\beta$ такое, что

$$
\begin{aligned}
& \frac{A_{1}}{100}+\beta B_{1} B_{2} B_{4} \sum_{n=0}^{\infty} \varkappa^{n}\left(\frac{1}{\sqrt{\alpha \lambda^{n}}}+B_{3}\right)<\frac{1}{10 C_{4}} B_{1} A_{1} \\
& \frac{B_{2}}{\sqrt{\lambda}} \frac{\beta}{\sqrt{\alpha}}<A_{2}, \quad \frac{B_{2}}{\sqrt{\lambda}} \frac{\beta}{\sqrt{\alpha}} \frac{B_{4}}{B_{1}}<A_{3}, \quad \frac{\beta B_{2} B_{3} B_{4}}{B_{1}}<A_{4}
\end{aligned}
$$

и

$$
\beta<\frac{1}{2} \alpha \varkappa^{2}
$$

где

$$
B_{3}=\sup _{\Lambda_{0}} \mathscr{V}\left(H_{0}\right)+1+\varepsilon \gg B_{1}, \quad B_{4}=2(1-\varepsilon) \sup _{n \leqslant K} \sup _{x \in \Delta_{n}}|\partial S|^{-1}>1 .
$$

Отметим, что требуемый порядок малости $\beta$ (как и сами числа $P, P_{1}$ ) будет еще уточняться в п. 2.6 при оценивании меры множеств $\mathfrak{D}_{n}$.

Найдем число $m_{1}>m_{0}$ такое, что для любого $n>m_{1}$

$$
y_{n}<\alpha \lambda^{P_{1}}, \quad\left|\Lambda_{n}\right|<\beta, \quad \mathscr{V}\left(H_{n}\right)<\frac{A_{1}}{100} .
$$

Выберем число $m_{2}>m_{1}$ такое, что для любых чисел $m$ и $n$, удовлетворяющих неравенствам $m>m_{2}, m_{1}>n>100$,

$$
y_{m}<\frac{1}{2} \alpha \lambda^{P_{1}}, \quad\left|\Lambda_{n}\right|<\frac{1}{3} \sum_{k=n+1}^{m-1}\left|\Lambda_{k}\right| .
$$

При изменении параметра $b$ числа $S(1), p, y_{n}, n=1, \ldots, N$ (а стало быть, и интервалы $\Lambda_{n}$ ), меняются. Соответствующие параметрические производные этих величин будем называть скоростями их движения. Первая оценка в утверждении (i) леммы 4 говорит о том, что критическое значение $S(1)$ движется с почти единичной скоростью. Наряду с этим оценка (2.15) означает, что при $n>m_{0}$ скорости интервалов $\Lambda_{n}$ малы (см. [2]). Сравнение этих скоростей показывает, что параметрический интервал вблизи $b=2$ разбивается на интервалы $\mathfrak{E}_{0}$ такие, что при изменении $b$ в пределах одного из $\mathfrak{E}_{0}=\mathfrak{E}_{0, M}$ критическое значение $S(1)$ пробегает определенный интервал $\Lambda_{n}=\Lambda_{M+1}$, где $M=M_{c}(b)$ введено в лемме 3 . Положим

$$
\mathfrak{D}_{0}=\bigcup_{M>m_{2}} \mathfrak{E}_{0, M}
$$


При этом интервалы $\mathfrak{E}_{0, M}, M>m_{2}$, называются составляющими интервалами множества $\mathfrak{D}_{0}$. Отметим, что мера $\mathfrak{D}_{0}$ оценивается равномерно по $c$.

Перейдем к определению отображения $F_{0}$. Прежде заметим, что в силу сказанного в начале этого пункта можно исключить из $\operatorname{dom}(\bar{T})$ множество малой меры так, что получившееся сужение будет удовлетворять свойству (vi) отображений $G_{n}$ при $n=0$. Аккуратная реализация этого приводит ниже к отображениям $F_{0}$ и $G_{0}$.

Зафиксируем какой-нибудь из интервалов $\mathfrak{E}_{0}=\left(b_{0}^{\prime}, b_{0}^{\prime \prime}\right)$ множества $\mathfrak{D}_{0}$ и обозначим соответствующий интервал изменения $S(1)$ через $\varkappa_{0}, \varkappa_{0}=\left(y_{M+1}, y_{M}\right)$, $M>m_{2}$. Найдутся числа $n_{0}>n_{-1}>\cdots>n_{-P}>\cdots>n_{-P-3}>m_{0}$ такие, что для любого $b \in \mathfrak{E}_{0}$ убьвающая последовательность интервалов $\lambda_{i}=\left(S(1), y_{n_{i}}\right)$, $i=-P-3, \ldots,-1,0$, подчинена условию

$$
\alpha \lambda^{i}<\left|\lambda_{i}\right|<2 \alpha \lambda^{i}
$$

Действительно, зафиксируем $i$ и $b=b_{0}^{\prime \prime}$ и положим $n_{i}=n-1$, где $n$ таково, что $\frac{3}{2} \alpha \lambda^{i} \in \Lambda_{n}, m_{1}>n>m_{0}$ (см. также п. 2.7). Оценка сверху на длину $\lambda_{i}$ вытекает из второго неравенства в $(2.21)$ и из сравнения скоростей $S(1)$ (лемма 4 ) и $y_{n_{i}}$ (оценка (2.15)). Оценка снизу выводится с помощью следуюшей леммы, которая в неявной форме в такой же ситуации использовалась в [2; с. 62].

Лемма 6. Пусть $\phi: \mathbb{R} \rightarrow \mathbb{R}, \psi: \mathbb{R} \rightarrow \mathbb{R}, \zeta: \mathbb{R} \rightarrow \mathbb{R}$ суть дифференцируемье функиии такие, что для некоторых малых чисел $\varepsilon_{1}>0, \varepsilon_{2}>0$ и для любого $x \in \mathbb{R}$

$$
1-\varepsilon_{1}<\left|\zeta^{\prime}(x)\right|<1+\varepsilon_{1}, \quad \max \left(\left|\phi^{\prime}(x)\right|,\left|\psi^{\prime}(x)\right|\right)<\varepsilon_{2}, \quad \phi(x)<\psi(x) .
$$

Если $x_{1}$ и $x_{2}$ задань уравнениями $\phi\left(x_{1}\right)=\zeta\left(x_{1}\right), \psi\left(x_{2}\right)=\zeta\left(x_{2}\right)$, то для любого $i=1,2$

$$
\left(1+\varepsilon_{1}+\varepsilon_{2}\right)^{-1}\left|\psi\left(x_{i}\right)-\phi\left(x_{i}\right)\right|<\left|x_{2}-x_{1}\right|<\left(1-\varepsilon_{1}-\varepsilon_{2}\right)^{-1}\left|\psi\left(x_{i}\right)-\phi\left(x_{i}\right)\right| .
$$

Последовательность $\lambda_{i}, i=-P-3, \ldots,-1,0$, порождает убывающую последовательность интервалов $\delta_{i}=S^{-1}\left(\lambda_{i}\right), i=-P-3, \ldots,-1,0$, экспоненциально сжимающихся к $x=1$. Пусть для любого $i$ символ $\delta_{i}^{-(+)}$обозначает левую (правую) компоненту связности множества $\delta_{i-1} \backslash \delta_{i}$. В силу оценок $(2.14)$ и $(2.22)$ получаем

$$
\frac{1}{10 \sqrt{\lambda}}<\frac{\left|\delta_{i}^{-(+)}\right|}{\left|\delta_{i}\right|}<\frac{1}{\sqrt{\lambda}}, \quad \frac{\left|\delta_{i}\right|}{\left|\delta_{i-1}\right|}<2 \sqrt{\lambda}
$$

и $\left|\Delta_{M+1}\right| \ll\left|\delta_{0}\right|<\left|\delta_{-P-3}\right| \ll|I|$.

Положим

$$
F_{0}=\left.\bar{H}\right|_{(S(1), p) \backslash\left(\lambda_{0} \cup \cup_{H_{0}}\left(H_{0}\right)^{-1}\left(\delta_{-P}\right)\right)}, \quad G_{0}=F_{0} \circ S .
$$

В дальнейшем интервал определения $J$ произвольного диффеоморфизма $F$ будем обозначать через $\Lambda F$, если $J \subset(0, p)$, и через $\Delta F$, если $J \subset I$. Кроме того, будем использовать следующие обозначения: символ $F_{0}^{0}$ - для ветвей отображения $F_{0}$, определенных внутри интервалов $\Lambda_{0}$, символ $F^{0}-$ для всех остальных ветвей $F_{0}$ и символ $Q_{0}$ - для диффеоморфизмов $H_{n}, n=m_{1}+1, \ldots, M, M+1$. Диффеоморфизмы $Q_{0}$ называются диффеоморфизмами глубиньл 0 , а интервалы $\Lambda Q_{0}-u н т е р-$ валами глубины 0. Отметим, что на последующих шагах индукции не делается 
композиций в какие-либо интервалы, расположенные вне интервалов глубины 0. Заметим, что производные диффеоморфизмов $F^{0}$ и $Q_{0}$ контролируются с помощью леммы 5, причем $\left|\Lambda Q_{0}\right|<\beta$ для любого $Q_{0}$. В принятых обозначениях имеем

$$
\mathfrak{k}_{0}=\left\{\Lambda: \Lambda=\Lambda F^{0}, \Lambda=\Lambda F_{0}^{0}\right\}
$$

Дополнение к множеству $\operatorname{dom}\left(F^{0}\right)$ в интервале $(S(1), p)$ организовано в виде объединения интервала $\lambda_{0}$ с прообразами $\left(H_{0}\right)^{-1}\left(\delta_{-P}\right)$, которые с этого момента будут называться $\lambda$-интервалами. Дополнение $\mathfrak{M}_{0} \mathrm{k} \mathrm{множеству} \operatorname{dom}\left(G^{0}\right)$ представляет собой объединение интервала $\delta_{0}$ с прообразами $\lambda$-интервалов под действием $S$. Все эти интервалы (вместе с $\delta_{0}$ ) назьваются $\delta$-интервалами иага 0 . Обозначим через $\mathfrak{m}_{0}$ множество $\delta$-интервалов шага 0 и через $\mathfrak{M}_{i}, i=1,2,3$, множества $\delta_{-i} \cup \bigcup_{T_{0}}\left(T_{0}\right)^{-1}\left(\delta_{-P-i}\right)$. Как $\lambda$-интервалы, так и $\delta$-интервалы отображаются с помощью определенных диффеоморфизмов на интервал $\delta_{-P}$ (кроме центрального $\delta_{0}$, который отображается на себя самого с помощью тождественного диффеоморфизма). Если $\xi$ является одним из $\lambda(\delta)$-интервалов и $f: \xi \rightarrow \delta_{i}, i=0,-P,-$ соответствующий диффеоморфизм, то $f$ продолжаем до диффеоморфизма $\widehat{f}$ на $\delta_{i-3}$. Введем обозначения: $\xi^{\prime}=\widehat{f}^{-1}\left(\delta_{i-1}\right), \xi^{\prime \prime}=\widehat{f}^{-1}\left(\delta_{i-2}\right), \xi^{\prime \prime \prime}=\widehat{f}^{-1}\left(\delta_{i-3}\right)$. В случае, когда $\xi$ есть $\lambda$-интервал, отметим, что ветви $F_{0}^{0}$, определенные внутри множества $\xi^{\prime} \backslash \xi$, находятся в биективном соответствии с ветвями отображения $G_{0}$, заданньми внутри множества $\delta_{-P-1} \backslash \delta_{-P}$. Обозначим через $\tau_{2}>1$ константу Кёбе, соответствуюшую паре интервалов $\delta_{i}, \delta_{i-1}$. Вследствие оценок $(2.23) \tau_{2}$ зависит только от $\lambda$. Кроме того,

$$
\bigcup_{\xi \in \mathfrak{m}_{0}} \xi^{\prime \prime \prime}=\mathfrak{M}_{-3} .
$$

Первый шаг конструкции. Сделаем композицию отображения $G_{0}$ во все критические интервалы, т.е. в $\Lambda_{M}$ и $\Lambda_{M+1}$. Полученные при этом диффееоморфизмы обозначим через $Q_{1}$. Эти диффеоморфизмы и их интервалы определения называются соответственно диффеоморфизмами и интервалами глубины 1 . Они соседствуют с прообразами $\delta$-интервалов. Наряду с имеющимися $\lambda$-интервалами эти прообразы также называются $\lambda$-интервалами и отображаются с помошюю соответствующих продолжаемых диффеоморфизмов на $\delta_{-P}$ и $\delta_{0}$. Изымем из $\varkappa_{0}$ множество $\left(H_{M+1}\right)^{-1} \mathfrak{M}_{-3}$. То, что осталось, представляет собой объединение $(\bmod 0)$ некоторых интервалов глубины 1. Совокупность этих интервалов обозначается через $\mathfrak{p}_{1}$. Покажем, что скорости движения интервалов $\Lambda Q_{1}$ малы по сравнению с единицей.

ЛЕмма 7. Для любого диффеоморфизма $Q_{1}$ выполнена оценка

$$
\mathscr{V}\left(Q_{1}\right)<\frac{A_{1}}{100}+\beta B_{1} B_{2} B_{4}\left(\frac{1}{\sqrt{\alpha}}+B_{3}\right)
$$

ДокАЗАТЕльство. Имеем $Q_{1}=F \circ S \circ Q_{0}$, где $F=F^{0}$ или $F=F_{0}^{0}$. Пользуясь формулой (2.7), запишем

$$
\frac{\partial_{b} Q_{1}}{\partial Q_{1}}=\frac{\partial_{b} F}{\partial F} \frac{1}{\partial S \partial Q_{0}}+\frac{\partial_{b} S}{\partial S} \frac{1}{\partial Q_{0}}+\frac{\partial_{b} Q_{0}}{\partial Q_{0}}
$$


поэтому надо оценить $\mathscr{V}\left(F_{0}^{0}\right)$. Так как $F_{0}^{0}=F^{0} \circ S \circ H_{0}, \Lambda F^{0} \subset\left(y_{n_{-P}}, y_{K}\right)$, оценим

$$
\mathscr{V}\left(F_{0}^{0}\right)<\mathscr{V}\left(F^{0}\right) \sup \left|\frac{1}{\partial S \partial H_{0}}\right|+\mathscr{V}\left(S, S^{-1}\left(\Lambda F^{0}\right)\right) \sup \left|\frac{1}{\partial H_{0}}\right|+\mathscr{V}\left(H_{0}\right)=\mathscr{B}_{1} .
$$

В соответствии с замечанием 8 имеем

$$
\mathscr{V}\left(S, S^{-1}\left(\Lambda F^{0}\right)\right) \leqslant(1+\varepsilon) \sup _{x: S(x)-S(1) \geqslant \alpha \lambda^{-P}} \frac{1}{|\partial S|} .
$$

Поэтому

$$
\begin{aligned}
\mathscr{B}_{1} & <B_{1} \frac{\sqrt{\lambda}^{P}}{2(1-\varepsilon) \sqrt{\alpha}} \frac{\tau C_{1}}{|I|}+B_{3}-1-\varepsilon \\
& <B_{1} B_{2} C_{1} \sqrt{\lambda}^{P} \frac{1}{\sqrt{\alpha}}+B_{3}-1-\varepsilon<\frac{1}{\sqrt{\alpha}}+B_{3}-1-\varepsilon
\end{aligned}
$$

(в этой выкладке использованы неравенства $(2.13),(2.14)$ и (2.22), а также продолжаемость $\left.H_{0}\right)$. Подставляя последнюю оценку в равенство $(2.26)$, запишем

$$
\mathscr{V}\left(Q_{1}\right)<\max \left(B_{1} B_{2} \frac{\beta}{\sqrt{\alpha}},\left(\frac{1}{\sqrt{\alpha}}+B_{3}\right) B_{2} \beta B_{4}\right)+\mathscr{V}\left(Q_{0}\right)
$$

откуда с учетом последнего из соотношений в (2.20) вытекает требуемое неравенство.

Отметим, что правая часть неравенства (2.25) не превосходит $10^{-1}$ вследствие условий $(2.16),(2.17)$. Отмеченное соотношение скоростей показывает, что внутри интервала $\mathfrak{E}_{0}$ находится множество, состоящее из интервалов $\mathfrak{E}_{1}$ и находящееся в биективном соответствии с $\mathfrak{p}_{1}$. Объединение интервалов $\mathfrak{E}_{1}$ есть часть множества $\mathfrak{D}_{1}$, расположенная в интервале $\mathfrak{E}_{0}$. Интервалы $\mathfrak{E}_{1}$ назьваются составляющими интервалами множества $\mathfrak{D}_{1}$.

Зафиксируем интервал $\mathfrak{E}_{1}$. Когда $b$ изменяется в пределах $\mathfrak{E}_{1}, S(1)$ пробегает определенный интервал $\varkappa_{1}$ вида $\Lambda Q_{1} \in \mathfrak{p}_{1}$, расположенный относительно далеко от $\lambda$-интервалов, как вытекает из включения (2.24). Заметим, что отображение $F_{1}$ уже определено на элементах множества $\mathfrak{k}_{0}$. Осталось определить его на части дополнения к $\operatorname{dom}\left(F_{0}\right)$ в интервале $(S(1), p)$. На первых шагах стратегия проста. Действуя полностью аналогично тому, как это делалось при построении отображения $F_{0}$, выберем интервал $\lambda_{1}=\left(S(1), y_{n_{1}}\right) \subset \lambda_{0}$ такой, что для любого $b \in \mathfrak{E}_{1} \alpha \lambda<\left|\lambda_{1}\right|<2 \alpha \lambda$, и определим

$$
\left.F_{1}\right|_{\lambda_{0} \backslash \lambda_{1}}=\bar{H}
$$

Ветви, составляющие $\left.F_{1}\right|_{\lambda_{0} \backslash \lambda_{1}}$, обозначим через $F_{1}^{1}$. Если $\xi$ есть произвольный $\lambda$-интервал вне $\lambda_{0}$ и $f: \xi \rightarrow \delta_{-P}-$ соответствующий диффеоморфизм, то положим

$$
\left.F_{1}\right|_{\xi \backslash \eta}=\bar{H}
$$

где $\eta=f^{-1}\left(\delta_{-P+1}\right) \subset \xi$. При этом $\xi$ перестает быть $\lambda$-интервалом, а им становится $\eta$, причем в силу оценок $(2.23)$ и продолжаемости $f$ имеем

$$
|\eta|<2 \tau_{2} \sqrt{\lambda}|\xi|
$$


Сделанная композиция назьвается заполнением $\lambda$-интервала $\xi$. Ветви всех отображений $\left.F_{1}\right|_{\xi \backslash \eta(\xi)}$ для всевозможных $\lambda$-интервалов $\xi$, расположенных вне $\lambda_{0}$, обозначаются через $F_{1}^{0}$. Таким образом,

$$
\mathfrak{k}_{1}=\mathfrak{k}_{0} \cup\left\{\Lambda: \Lambda=\Lambda F_{1}^{1}, \Lambda=\Lambda F_{1}^{0}\right\} .
$$

Определим $\delta_{1}=S^{-1}\left(\lambda_{1}\right), G_{1}=F_{1} \circ S$. Заметим, что после сделанной на этом шаге композиции критическими будут только интервалы глубины 1 . Согласно предварительной конструкции их длины не превосходят $\beta \varkappa$. Если $\Lambda$ представляет собой один из таких интервалов, то либо $\Lambda \subset \varkappa_{0}$, либо $\Lambda \subset \Lambda_{M}$. Таким образом, критические диффеоморфизмы будут определены очень близко от $S(1)$ и заведомо внутри $\lambda_{1}$. Отсюда следует, что отображение $G_{1}$ удовлетворяет требованию (vi).

Дополнение к $\operatorname{dom}\left(G_{1}\right)$ представляет собой объединение $\delta$-интервалов шага 1 , образующих множество $\mathfrak{m}_{1}$. Все эти интервалы, кроме центрального $\delta_{1}$, являются прообразами новых $\lambda$-интервалов под действием $S$. Суммируя неравенства $(2.27)$ по всем $\delta$-интервалам шага 0 и используя $(2.23)$ при $i=1$, получаем

$$
\left|\bigcup_{\delta \in \mathfrak{m}_{1}} \delta\right|<2 \tau_{2} \sqrt{\lambda}\left|\bigcup_{\delta \in \mathfrak{m}_{0}} \delta\right|
$$

Будем считать, что

$$
10 \tau_{2}^{2} \sqrt{\lambda}<\frac{1}{4}
$$

основьваясь на оценке $\tau_{2}<\exp \left(\nu\left(10^{-1} \sqrt{\lambda}\right) 2 \sqrt{\lambda}\right)$ (см. п. 2.1) и уменьшая $\lambda$ в случае необходимости.

Заметим, что отображения $F_{1}$ и $G_{1}$ зависят непрерывно от $b$ на интервале $\mathfrak{E}_{1}$.

$(n+1)$-й шаг индукции. На $n$-м шаге построено множество $\mathfrak{D}_{n}$, которое удовлетворяет предъявляемым к нему требованиям (vii), (ix), и зафиксирован составляющий интервал $\mathfrak{E}_{n}$ множества $\mathfrak{D}_{n}$. При этом существует интервал $\varkappa_{n}=\left(y_{n}^{\prime}, y_{n}^{\prime \prime}\right)$ глубины $n$ такой, что при изменении $b$ в пределах $\mathfrak{E}_{n}$ критическое значение $S(1)$ пробегает интервал $\varkappa_{n}$; обозначим через $R_{n}: \varkappa_{n} \rightarrow I$ соответствуюший диффеоморфизм глубины $n$. Продолжаемый диффеоморфизм называется диффеоморфизмом глубинь $k, k \leqslant n$, если он получен в результате композиции отображения $G_{k-1}$ в интервал глубины $k-1$. Каждый из диффеоморфизмов глубины $k$ обозначается символом $Q_{k}$. Любой интервал вида $\Lambda Q_{k}$ называется интервалом глубины $k$. Для каждого $k$ и для любого интервала $\Lambda Q_{k}$ вьполнена оценка $\left|\Lambda Q_{k}\right|<\beta \varkappa^{k}$, что вытекает из продолжаемости $Q_{k}$ и свойств предварительной конструкции. Отметим, что отображения $F_{n}$ и $G_{n}$ меняются непрерьвно на интервале $\mathfrak{E}_{n}$.

В дополнение к требованиям (i)-(vi) множества $\mathfrak{k}_{n}, \mathfrak{l}_{n}$ и отображения $F_{n}, G_{n}$ обладают рядом свойств, которые будут формулироваться постепенно по мере надобности для обоснования построений на шаге $n+1$. Приведем ряд свойств, относящихся к геометрии диффеоморфизмов, введенных в рассмотрение за первые $n$ шагов:

$\left(b_{1}\right)$ каждая из ветвей отображения $F_{n}$ относится к одному из следующих типов: ветви $H_{i}, m_{1} \geqslant i>K$, ветви $Q_{l}, 0 \leqslant l \leqslant n$, ветви, полученные на шагах $0,1, \ldots, n$ в результате заполнений; в этой связи отметим, что ветви $F_{0}^{0}$, созданные на этапе предварительной конструкции, с точки зрения оценок удобнее относить к третьему типу; 
$\left(b_{2}\right)$ дополнение к $\operatorname{dom}\left(F_{n}\right)$ в множестве $(S(1), p)$ представляет собой объединение некоторого интервала $\lambda_{n}$ вида $\left(S(1), \widetilde{y}_{n}\right)$ с дизъюнктными интервалами, которые расположены вне $\lambda_{n}$ и называются $\lambda$-интервалами; как результат, дополнение к множеству $\operatorname{dom}\left(G_{n}\right)$ в $I$ является дизъюнктным объединением интервалов, среди которых находится и $\delta_{n}=S^{-1}\left(\lambda_{n}\right)$ и которые называются $\delta$-интервалами шага $n$; при этом для любого $b \in \mathfrak{E}_{n}$ выполнены оценки (2.22) и (2.23) с $i=n$;

$\left(b_{3}\right)$ интервал $\lambda_{n}$, вообще говоря, разбивается $(\bmod 0)$ на некоторые интервалы глубин $l, l \in\{0,1, \ldots, n\}$, интервал $\varkappa_{n} \cap S(I)$, некоторые интервалы $\Lambda_{k}$, $k \in\left\{n_{0}, \ldots, m_{1}\right\}$ (для достаточно малых $n$ ), и прообразы $\delta$-интервалов шагов $0,1, \ldots, n-1$; эти прообразы тоже называются $\lambda$-интервалами, все остальные из перечисленных интервалов (вместе с $\varkappa_{n}$ ) образуют множество $\mathfrak{n}_{n}$, и совокупность соответствуюших продолжаемых диффеоморфизмов (вместе с $R_{n}$ ) задает отображение $Z_{n}: \bigcup_{\Lambda \in \mathfrak{n}_{n}} \Lambda \rightarrow I$; все критические интервалы являются интервалами глубины $n$.

Сформулируем лемму, с помощью которой контролируются скорости интервалов из множеств $\mathfrak{k}_{n}$ и $\mathfrak{n}_{n}$.

Лемма $8(n)$. Справедливь следующие оценки:

(i) для любого $k \in\{0, \ldots, n\}$

$$
\sup _{Q_{k}} \mathscr{V}\left(Q_{k}\right)<\frac{A_{1}}{100}+\beta B_{1} B_{2} B_{4} \sum_{i=0}^{k-1} \varkappa^{i}\left(\frac{1}{\sqrt{\alpha \lambda^{i}}}+B_{3}\right)
$$

(ii) для любого $k \in\{1, \ldots, n\}$ и для каждой ветви $F$ отображсения $F_{n}$ такой, что $\Lambda F \subset \lambda_{k-1} \backslash \lambda_{k}$,

$$
\mathscr{V}(F)<\frac{B_{1}}{\sqrt{\lambda^{n-k}}}-1-\varepsilon ;
$$

(iii) для любой ветви $F$ отображения $F_{n}$ такой, что $\Lambda F \subset \bigcup_{H_{0}} \operatorname{dom}\left(H_{0}\right)$,

$$
\mathscr{V}(F)<\frac{1}{\sqrt{\alpha \lambda^{n}}}+B_{3}-1-\varepsilon .
$$

Обозначим через $\mathfrak{m}_{n}$ множество $\delta$-интервалов шага $n, \mathfrak{M}_{n}=\bigcup_{\xi \in \mathfrak{m}_{n}} \xi$, и перейдем к описанию шага $n+1$. Сделаем композицию отображения $G_{n}$ во все критические интервалы. Полученные при этом диффеоморфизмы и их интервалы определения называются соответственно диффеоморфизмами и интервалами глубины $n+1$. Они обозначаются через $Q_{n+1}$ и $\Lambda Q_{n+1}$ соответственно. Согласно предварительной конструкции для любого диффеоморфизма $Q_{n+1}$ имеем $\left|\Lambda Q_{n+1}\right|<\beta \varkappa^{n+1}$. По соседству с интервалами глубины $n+1$ расположены прообразы элементов множества $\mathfrak{m}_{n}$, которые тоже причисляются к $\lambda$-интервалам. Изымем из интервала $\varkappa_{n}$ множество $\left(R_{n}\right)^{-1}\left(\mathfrak{M}_{n-3}\right)$. То, что осталось, представляет собой некоторое семейство интервалов глубины $n+1$, которое обозначим через $\mathfrak{p}_{n+1}$.

Заметим, что критические интервалы в совокупности имеют сравнительно малую меру.

$\left(b_{4 n}\right)$ Пусть $J$ есть минимальный интервал, содержащий все критические интервалы шага $n$. Тогда $|J|<\beta \varkappa^{n-1}$. 
В самом деле, либо все критические интервалы содержатся внутри $\varkappa_{n-1}$, либо есть интервалы с этим свойством вне $\varkappa_{n-1}$. Обозначим их через $J_{1}, \ldots, J_{i}$. В первом случае оценка очевидна. Во втором случае выбор параметра предварительной конструкции означает, что $\operatorname{dist}\left(S(1), J_{j}\right)<10^{-1} \beta \varkappa^{n-1}$ для любого $j \in\{1, \ldots, i\}$. Это вытекает из того, что в силу свойства $\left(b_{3}\right) J_{j}$ является интервалом глубины $n$, стало быть, содержится в некотором интервале глубины $n-1$, причем должен примькать к его левой гранище. Нетрудно видеть, что последнее неравенство влечет свойство $\left(b_{4 n}\right)$.

Таким образом, интервалы глубины $n+1$, создаваемые на этом шаге, сосредоточены в $\beta \varkappa^{n-1}$-окрестности критического значения $S(1)$. Покажем, что интервалы $\Lambda Q_{n+1}$ двигаются медленно по сравнению с критическим значением $S(1)$. Принимая во внимание неравенство (2.17), для этого достаточно доказать соответствующие оценки леммы $8(n+1)$.

ДОКАЗАТЕЛЬСТво УТВЕРЖ ДЕНИЯ (i) ЛЕМмы $8(n+1)$. Пусть $Q_{n+1}$ - некоторый диффеоморфизм глубины $n+1$. Тогда $Q_{n+1}=F \circ S \circ Q_{n}$, где $Q_{n}$ - определенный диффеоморфизм глубины $n$, а $F$ - одна из ветвей отображения $F_{n}$. Используя формулу (2.7), запишем

$$
\mathscr{V}\left(Q_{n+1}\right)<\mathscr{V}(F) \sup \frac{1}{\partial S \partial Q_{n}}+\mathscr{V}\left(S, S^{-1}(\Lambda F)\right) \sup \frac{1}{\partial Q_{n}}+\mathscr{V}\left(Q_{n}\right)
$$

Предположим, что $\Lambda F \subset \lambda_{k-1} \backslash \lambda_{k}$ для некоторого $k \in\{1, \ldots, n\}$. Учитывая оценку (ii) леммы $8(n)$, продолжаемость $Q_{n}$, неравенства $(2.14),(2.22)$ и замечание 8 , оценим

$$
\begin{aligned}
\mathscr{V}\left(Q_{n+1}\right) & <(\mathscr{V}(F)+1+\varepsilon) \frac{1}{2(1-\varepsilon) \sqrt{\alpha \lambda^{k}}} \frac{\tau}{|I|}\left|\Lambda Q_{n}\right|+\mathscr{V}\left(Q_{n}\right) \\
& <\frac{B_{1}}{\sqrt{\lambda^{n-k}}} B_{2} \frac{\beta \varkappa^{n}}{\sqrt{\alpha \lambda^{k}}}+\mathscr{V}\left(Q_{n}\right) .
\end{aligned}
$$

Если $\Lambda F \subset \bigcup_{\Lambda_{0}} \Lambda_{0}$, то, действуя аналогично, получаем

$$
\begin{aligned}
\mathscr{V}\left(Q_{n+1}\right) & <(\mathscr{V}(F)+1+\varepsilon) \frac{B_{4}}{2(1-\varepsilon)} \frac{\tau}{|I|}\left|\Lambda Q_{n}\right|+\mathscr{V}\left(Q_{n}\right) \\
& <\left(\frac{1}{\sqrt{\alpha \lambda^{n}}}+B_{3}\right) B_{2} B_{4} \beta \varkappa^{n}+\mathscr{V}\left(Q_{n}\right) .
\end{aligned}
$$

Серии неравенств (2.31) и (2.32) влекут требуемую оценку.

ЗАмЕчАнИЕ 9. В формулировке леммы 8 и в только что приведенных выкладках не сказано ни слова о ветвях отображения $F_{n}$ вида $F^{0}$. Ввиду леммы 5 для таких ветвей оценки аналогичны.

Отмеченное сравнение скоростей означает, что внутри интервала $\mathfrak{E}_{n}$ существует множество, состоящее из интервалов $\mathfrak{E}_{n+1}$ и находящееся в биективном соответствии с множеством $\mathfrak{p}_{n+1}$. Объединение интервалов $\mathfrak{E}_{n+1}$ есть часть множества $\mathfrak{D}_{n+1}$, расположенная в интервале $\mathfrak{E}_{n}$. Интервалы $\mathfrak{E}_{n+1}$ называются составляющими интервалами множества $\mathfrak{D}_{n+1}$.

Зафиксируем интервал $\mathfrak{E}_{n+1}=\left(b_{n+1}^{\prime}, b_{n+1}^{\prime \prime}\right)$. Когда $b$ меняется в пределах $\mathfrak{E}_{n+1}$, критическое значение $S(1)$ остается относительно далеко от $\lambda$-интервалов (см. 
свойство $\left.\left(h_{4 n}\right)\right)$ и пробегает при этом определенный интервал $\varkappa_{n+1}$ глубины $n+1$ из множества $\mathfrak{p}_{n+1}$.

Так как отображение $F_{n+1}$ уже определено на элементах множества $\mathfrak{k}_{n}$, осталось его задать внутри интервала $\lambda_{n}$ и $\lambda$-интервалов, лежаших вне $\lambda_{n}$. Выберем интервал $\lambda_{n+1}$ вида $\left(S(1), \widetilde{y}_{n+1}\right)$, удовлетворяющий оценке $(2.22)$ с $i=n+1$ для любого $b \in \mathfrak{E}_{n+1}$. Делается это на основании следующих свойств $\lambda$-интервалов (подчеркнем, что речь идет о $\lambda$-интервалах, фигурирующих в конструкции перед шагом $n+1)$ :

$\left(h_{1 n}\right)$ если $\xi$ является одним из $\lambda$-интервалов, то $\xi$ отображается с помощью динамически заданного (т.е. являюшегося итерацией отображения $S$ ) диффеоморфизма $f$ на определенный интервал $\delta_{l}, l \leqslant n-1$, причем $f$ продолжаем до диффеоморфизма на интервал $\delta_{l-3}$ и, стало быть, определены интервалы $\xi^{\prime} \subset \xi^{\prime \prime} \subset \xi^{\prime \prime \prime}$ (см. обсуждение стартового шага конструкции);

$\left(h_{2 n}\right)$ для любого $\lambda$-интервала $\xi$ такого, что $\xi \cap(S(1), p) \neq \varnothing$, имеем включение $\xi^{\prime \prime \prime} \subset(S(1), p) ;$

$\left(h_{3 n}\right)$ для любых двух $\lambda$-интервалов $\xi_{1}$ и $\xi_{2}$ либо $\xi_{1}^{\prime} \cap \xi_{2}^{\prime}=\varnothing$, либо один из интервалов $\xi_{1}^{\prime}, \xi_{2}^{\prime}$ содержится в другом; если $\xi$ является одним из $\lambda$-интервалов, то $\xi^{\prime} \subset \lambda_{n}$, как только $\xi^{\prime} \cap \lambda_{n} \neq \varnothing$.

Зафиксируем $b=b_{n+1}^{\prime \prime}$, положим $y_{n+1}^{\prime}=\frac{3}{2} \alpha \lambda^{n+1}$ и рассмотрим интервал $\left(S(1), S(1)+y_{n+1}^{\prime}\right)$. Заметим, что справа от точки $S(1)+y_{n+1}^{\prime}$ не может оказаться ни интервалов глубины $n+1$, ни прообразов $\delta$-интервалов шага $n$ (свойство $\left.\left(b_{4 n}\right)\right)$. Сушествуют две возможности: или $S(1)+y_{n+1}^{\prime} \in \xi^{\prime}$ для некоторого $\lambda$-интервала $\xi$, или $S(1)+y_{n+1}^{\prime} \in \Lambda Q_{l}, 0 \leqslant l \leqslant n$, причем согласно свойству $\left(h_{3 n}\right)$ можно считать, что интервал $\xi^{\prime}$ является максимальным. В первом случае в качестве $\widetilde{y}_{n+1}$ возьмем правую граничную точку $\xi^{\prime}$. Вследствие свойства $\left(h_{2 n}\right)$ имеем оценку $\left|\left(\xi^{\prime \prime} \backslash \xi^{\prime}\right)^{-}\right|<\frac{3}{2} \alpha \lambda^{n+1}$, где $\left(\xi^{\prime \prime} \backslash \xi^{\prime}\right)^{-}$обозначает левую компоненту связности множества $\xi^{\prime \prime} \backslash \xi^{\prime}$. Принимая во внимание первое из неравенств в $(2.23)$ и (2.30), запишем

$$
\begin{aligned}
\frac{3}{2} \alpha \lambda^{n+1}+\left|\xi^{\prime}\right| & =\frac{3}{2} \alpha \lambda^{n+1}+\left|\left(\xi^{\prime \prime} \backslash \xi^{\prime}\right)^{-}\right| \frac{\left|\xi^{\prime}\right|}{\left|\left(\xi^{\prime \prime} \backslash \xi^{\prime}\right)^{-}\right|} \\
& <\frac{3}{2} \alpha \lambda^{n+1}+10 \sqrt{\lambda} \tau_{2} \frac{3}{2} \alpha \lambda^{n+1}<2 \alpha \lambda^{n+1}
\end{aligned}
$$

Во втором случае в качестве $\widetilde{y}_{n+1}$ возьмем правую граничную точку интервала $\Lambda=\Lambda Q_{l}$. Предположим, что $l \geqslant[(n+1) \omega]-1$, где $\omega=\log \lambda(\log \varkappa)^{-1}$ и символ $[x]$ обозначает целую часть числа $x$. Тогда $|\Lambda|<2^{-1} \alpha \lambda^{n+1}$, что проверяется непосредственно с использованием оценки (2.19) и неравенства $|\Lambda|<\beta \varkappa^{l}$. Поэтому $\frac{3}{2} \alpha \lambda^{n+1}+|\Lambda| \leqslant 2 \alpha \lambda^{n+1}$, что и требовалось. Нетрудно видеть, что свойства $\left(h_{1 n}\right)-\left(h_{3 n}\right)$ при композиции в любой интервал, кроме $\varkappa_{n}$, выживают. Если $l<[(n+1) \omega]-1$, то сделаем композицию отображения $G_{l}$ в интервал $\Lambda$, после чего снова оказываемся в рамках двух возможностей и т. д. Таким образом, после конечного числа композиций удается осуществить выбор интервала $\lambda_{n+1}$, состоящего целиком из элементов множества $\mathfrak{n}_{n}$ и $\lambda$-интервалов, а также удовлетворяющего верхней оценке в (2.22). Проверка нижней оценки осуществляется с помощью леммы 6, условия (2.15) и(или) утверждения (i) леммы 8 и неравенства $\varkappa_{n+1}<\beta \varkappa^{n+1}$ в точности так же, как при выборе интервала $\lambda_{0}$. Отметим, что каждая из упомянутых композиций сопровождается соответствуюшей модификацией множества $\mathfrak{n}_{n}$ и отображения $Z_{n}$. 
Прежде чем перейти к определению отображения $F_{n+1}$, введем некоторые обозначения. Как было отмечено, ветви отображения $F_{n}$ различаются как по времени их создания, так и по расположению их интервалов определения. Это отражено в обозначениях. Для любых $k \in\{1, \ldots, n\}, p \in\{k, \ldots, n\}$ через $F_{p}^{k}$ обозначается каждая из ветвей отображения $F_{n}$, заданных внутри множества $\lambda_{k-1} \backslash \lambda_{k}$ и построенных на шаге $p$ (т.е. определенных на интервалах множества $\mathfrak{k}_{p} \backslash \mathfrak{k}_{p-1}$ ). Для любого $p \in\{0,1, \ldots, n\}$ символом $F_{p}^{0}$ обозначается каждая из ветвей, определенных внутри интервалов $\Lambda_{0}$ и созданных (в результате заполнения) на шаге $p$. С учетом этих замечаний имеем

$$
\mathfrak{k}_{n}=\left\{\Lambda: \Lambda=\Lambda F^{0}, \Lambda=\Lambda F_{p}^{k}, k=0,1, \ldots, n, p=k, \ldots, n\right\}
$$

Отметим, что каждая ветвь отображения $F_{n}$ вида $Q_{l}, 1 \leqslant l \leqslant n$, появилась в результате взятия композиции либо на шаге $l$, либо позднее, скажем, на шаге $k, k \gg l$, при выборе интервала $\lambda_{k}$. В первом случае $\Lambda Q_{l} \subset\left(\Lambda_{M+1} \cup \Lambda_{M}\right) \cap S(I)$, во втором случае $\Lambda Q_{l} \subset \lambda_{k-1}$, поэтому всегда $\Lambda Q_{l} \subset \lambda_{P_{2}}$ для некоторого $P_{2} \geqslant P_{1}$. Действительно, благодаря выбору начальных параметров $\left(\Lambda_{M+1} \cup \Lambda_{M}\right) \cap S(I) \subset \lambda_{P_{1}}$; по этой же причине на шагах $i, i=1,2, \ldots, P_{1}$, интервал $\lambda_{i}$ строится без участия композиций, поэтому $k>P_{1}$. Из сказанного вытекает, что на всех шагах конструкции все $\lambda$-интервалы (а стало быть, и ветви, получаемые с помощью заполнений) расположены либо внутри интервалов $\Lambda_{0}$, либо внутри $\lambda_{P_{2}}$. Считаем, что число $P_{2}$ с такими свойствами выбрано максимальньм.

В соответствии с введенной символикой будем обозначать через $F_{n+1}^{n+1}$ каждую из ветвей отображения $Z_{n}$, определенную внутри множества $\lambda_{n} \backslash \lambda_{n+1}$. Пусть $\xi$ представляет собой один из $\lambda$-интервалов, лежащих внутри множества $\lambda_{k-1} \backslash \lambda_{k}$, $k \in\left\{P_{2}+1, \ldots, n+1\right\}$, и $f: \xi \rightarrow \delta_{t}$ - соответствуюший диффеоморфизм, $t \leqslant n-1$. Сделаем заполнение интервала $\xi$, т.е. рассмотрим всевозможные композиции вида $R \circ f$, где $R$ является ветвью отображения $G_{t+1}$, определенной внутри множества $\delta_{t} \backslash \delta_{t+1}$. При этом $\xi$ перестает быть $\lambda$-интервалом, а ими становятся прообразы $f^{-1}(\eta) \subset \xi \delta$-интервалов $\eta$ шага $t+1$, расположенных внутри $\delta_{t}$. Каждая из упомянутых композиций для всевозможных $\xi \subset \lambda_{k-1} \backslash \lambda_{k}$ будет обозначаться через $F_{n+1}^{k}$. Полностью аналогично предыдушему сделаем заполнение всех $\lambda$-интервалов, лежащих внутри объединения всех $\Lambda_{0}$, и каждую из полученных ветвей обозначим через $F_{n+1}^{0}$.

Добавив диффеоморфизмы $F_{n+1}^{k}, k=0, P_{2}+1, \ldots, n+1$, к имеющимся ветвям отображения $F_{n}$, получим отображение $F_{n+1}$. Таким образом,

$$
\mathfrak{k}_{n+1}=\mathfrak{k}_{n} \cup\left\{\Lambda: \Lambda=\Lambda F_{n+1}^{k}, k=0, P_{2}+1, \ldots, n+1\right\}
$$

Покажем, что построенные на этом шаге ветви накрываемы. Простые выгисления, основанные на свойстве $\left(b_{4 n}\right)$ и условии $(2.19)$, говорят о том, что все ветви отображения $Z_{n}$, определенные внутри множества $\lambda_{n} \backslash \lambda_{n+1}$, накрываемы. Обратимся к ветвям, полученньм в результате заполнений. Рассмотрим одну из таких ветвей $F=F_{n+1}^{k}: \Lambda \rightarrow I$. Напомним, что $G_{k}=F_{k} \circ S$ для любого $k \in\{0,1, \ldots, n\}$, и предположим, что ветви отображения $F_{n}$ в окрестности $\lambda_{n}$ обладают следуюшим свойством:

$\left(b_{5 n}\right)$ для любой ветви $F_{n}^{n}: J \rightarrow I$ отображения $F_{n}$ интервал $\widehat{J}$ содержится в $\lambda_{n-2}$. 
Заметим, что $F=F_{t+1}^{t+1} \circ S \circ f$ для некоторых $t \leqslant n-1$ и $f$, где диффеоморфизм $f$ отображает $\lambda$-интервал $\xi$ на интервал $\delta_{t}$ и при этом продолжается до диффеоморфизма на $\delta_{t-3}$. Стало быть, ветвь $F$ накрываема в соответствии со свойствами $\left(b_{5(t+1)}\right)$ и $\left(h_{2 n}\right)$, а также со свойством накрьваемости $F_{t+1}^{t+1}$, причем $\widehat{\Lambda} \subset \xi^{\prime}$. Предположим, что $k=n+1$. Тогда $\xi^{\prime} \subset \lambda_{n} \backslash \lambda_{n+1}$, откуда делаем вывод, что для $F$ выполнено и условие $\left(b_{5}(n+1)\right)$.

Положим $G_{n+1}=F_{n+1} \circ S, \delta_{n+1}=S^{-1}\left(\lambda_{n+1}\right)$. Из сказанного ясно, что отображение $G_{n+1}$ удовлетворяет требованию (vi). Дополнение к $\operatorname{dom}\left(G_{n+1}\right)$ представляет собой объединение $\delta$-интервалов шага $n+1$, образующих множество $\mathfrak{m}_{n+1}$. Все эти интервалы, кроме центрального $\delta_{n+1}$, являются прообразами новых $\lambda$-интервалов под действием $S$. Интервал $\delta_{n+1}$ удовлетворяет оценкам (2.23) c $i=n+1$.

Оставшаяся часть этого пункта посвящена приготовлениям к шагу $n+2$. Проверим, что построенные на шаге $n+1$ ветви отображения $F_{n+1}$ удовлетворяют соответствующим оценкам леммы $8(n+1)$.

ДОКАЗАТЕЛЬСТВО УТВЕРЖДЕНИЙ (ii) И (iii) ЛЕМмы $8(n+1)$. (iii) Зафиксируем какую-либо из ветвей $F_{n+1}^{0}$. Найдется такой диффеоморфизм $H_{0}$, что $F_{n+1}^{0}=$ $F \circ S \circ H_{0}$, где $F$ представляет собой одну из ветвей $F_{n+1-P}^{t}, t \in\left\{\min \left(P_{2}+1\right.\right.$, $n+1-P), \ldots, n+1-P\}$. Это можно проверить по индукции, стартуя, к примеру, с $n=P$. Используя формулу (2.7), оценку (2.22), условие (2.13) и утверждение (ii) леммы $8(n)$, запишем

$$
\begin{aligned}
\mathscr{V}\left(F_{n+1}^{0}\right) & <(\mathscr{V}(F)+1+\varepsilon) \frac{1}{2(1-\varepsilon) \sqrt{\alpha \lambda^{t}}} \frac{\tau}{|I|} C_{1}+B_{3}-1-\varepsilon \\
& <\frac{B_{1}}{\sqrt{\lambda^{n+1-P-t}}} B_{2} C_{1} \frac{1}{\sqrt{\alpha \lambda^{t}}}+B_{3}-1-\varepsilon<\frac{1}{\sqrt{\alpha \lambda^{n+1}}}+B_{3}-1-\varepsilon
\end{aligned}
$$

что и требовалось доказать.

(ii) В соответствии с предположениями индукции $\left(b_{1}\right)$ и $\left(b_{3}\right)$ глубины диффеоморфизмов, построенных после шага $n$, подчинены следующим ограничениям:

$\left(b_{6}\right)$ предположим, что диффеоморфизм $Q_{l}, 1 \leqslant l \leqslant n$, является ветвью одного из отображений $F_{n}$ или $Z_{n}$ и $\Lambda Q_{l} \subset \lambda_{k-1} \backslash \lambda_{k}, k \in\left\{P_{2}+1, \ldots, n+1\right\}$; тогда $l \leqslant[\omega k]$.

Поясним это. Не ограничивая общности (см. обсуждение выбора интервала $\left.\lambda_{n+1}\right)$, можно считать, что $Q_{l}$ был создан на шаге $l$ в результате композиции в критический интервал глубины $l-1$. Принимая во внимание оценку в $\left(b_{4(l-1)}\right)$ и неравенство (2.22), получаем, что $\alpha \lambda^{k}<\beta \varkappa^{l-2}$. Учитывая условие (2.19), отсюда непосредственно выводим оценку на $l$.

Рассмотрим одну из ветвей $F=F_{n+1}^{k}, k \in\left\{\min \left(P_{2}+1, n+1\right), \ldots, n+1\right\}$. Сушествуют три возможности: либо $F=H_{m}, m \in\left\{n_{0}, \ldots, M-1\right\}$, либо $F=Q_{l}$, либо $F$ получено в результате заполнения некоторого $\lambda$-интервала $\xi$. В первом случае требуемая оценка обеспечивается леммой 5 . Во втором случае вследствие леммы $8(n)$ и неравенств $(2.16),(2.17)$ получаем $\mathscr{V}(F)<B_{1} A_{1}<B_{1} \lambda^{-\frac{n-k}{2}}-1-\varepsilon$.

Предположим, что ветвь $F$ возникла в результате заполнения интервала $\xi$. Од-

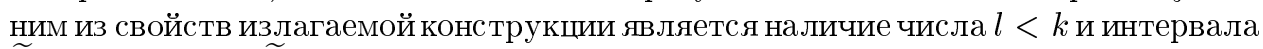
$\widetilde{\xi} \supset \xi$ таких, что $\widetilde{\xi}$ появился как прообраз $\delta$-интервала шага $l-1$ в результате композиции отображения $G_{l-1}$ в определенньй интервал глубины $l-1$. Основьваясь на этом, с помощью индукции по $n \geqslant k$ при фиксированном $k$ можно проверить, что 
найдется диффеоморфизм $Q_{l-1}$ такой, что $F_{n+1}^{k}=U \circ S \circ Q_{l-1}$, где $U$ представляет собой одну из ветвей $F_{n+1-k+l}^{t}, t \in\left\{0, \min \left(P_{2}+1, n+1-k+l\right), \ldots, n+1-k+l\right\}$. Отметим, что в силу $\left(b_{6}\right) n+1-k+l<n+1$, и мы можем использовать оценки леммы $8(n)$.

В случае $t=0$ оценим

$$
\begin{aligned}
\mathscr{V}(F) & <(\mathscr{V}(U)+1+\varepsilon) \frac{B_{4}}{2(1-\varepsilon)} \frac{\tau}{|I|}\left|\Lambda Q_{l-1}\right|+\mathscr{V}\left(Q_{l-1}\right) \\
& <\left(\frac{1}{\sqrt{\alpha \lambda^{n+1-k+l}}}+B_{3}\right) B_{2} B_{4} \beta \varkappa^{l-1}+B_{1} A_{1}=\mathscr{B}_{2} .
\end{aligned}
$$

С учетом (2.18) неравенство

$$
\mathscr{B}_{2}<\frac{B_{1}}{\sqrt{\lambda^{n+1-k}}}-1-\varepsilon
$$

вытекает из соотношения $B_{1}\left(A_{1}+A_{3}+A_{4}\right)<C_{4}$ (см. (2.16)).

Если $t \neq 0$, то тем же способом (опираясь при этом на оценку $(2.22)$ ) получаем

$$
\begin{aligned}
\mathscr{V}(F) & <(\mathscr{V}(U)+1+\varepsilon) \frac{1}{2(1-\varepsilon) \sqrt{\alpha \lambda^{t}}} \frac{\tau}{|I|}\left|\Lambda Q_{l-1}\right|+\mathscr{V}\left(Q_{l-1}\right) \\
& <\frac{B_{1}}{\sqrt{\lambda^{n+1-k+l-t}}} \frac{B_{2} \beta}{\sqrt{\alpha \lambda^{t}}} \varkappa^{l-1}+B_{1} A_{1}<\frac{B_{1}}{\sqrt{\lambda^{n+1-k}}}-1-\varepsilon,
\end{aligned}
$$

что и требовалось доказать.

ДОкАЗАТЕЛЬСтво СвоЙСтв $\left(b_{5(n+1)}\right),\left(h_{1(n+1)}\right)-\left(h_{3(n+1)}\right)$.

$\left(b_{5(n+1)}\right)$ Диффеоморфизмы $F_{n+1}^{n+1}$, не являющиеся ветвями отображения $Z_{n}$, уже обсуждались. Пусть $F=F_{n+1}^{n+1}$ есть ветвь $Z_{n}$, не удовлетворяющая требованию $\left(b_{5}(n+1)\right), l$ - глубина $F, l \ll n$. Сделаем композицию отображения $G_{l}$ в $\Lambda F$. Каждую из образовавшихся при этом ветвей обозначим также через $F_{n+1}^{n+1}$. Благодаря сделанному выбору параметров предварительной конструкции новые ветви $F_{n+1}^{n+1}$ будут обладать свойством $\left(b_{5(n+1)}\right)$. Отметим, что с этого момента символ $F_{n+1}^{n+1}$ не используется для обозначения $F$.

$\left(h_{3(n+1)}\right)$ Введем следуюшие обозначения. Пусть $\xi$-интервал, $f: \xi \rightarrow \delta_{t}-$ диффеоморфизм. Положим $\xi_{p}=\xi_{p}(\xi)=f^{-1}\left(\delta_{t+1}\right)$ и для каждого $\delta$-интервала $\eta$ шага $t+1$, отличного от $\delta_{t+1}$ и такого, что $\eta \subset \delta_{t}$, обозначим через $\xi_{s}=\xi_{s}(\xi)$ прообраз $f^{-1}(\eta)$.

Рассмотрим $\lambda$-интервалы, которые заполнялись на шаге $n+1$. Подчеркнем, что они не пересекаются и обладают свойством $\left(h_{3 n}\right)$. Зафиксируем один из таких интервалов $\xi$, отображаемый с помощью диффеоморфизма $f$ на, скажем, интервал $\delta_{l-1}, l \leqslant n$. На этапе заполнения $\xi$ образовались новые $\lambda$-интервалы $\xi_{p}$ и $\xi_{s}$. Очевидно, интервал $\xi_{p}^{\prime}$ определен и равен $\xi$. Согласно свойству $\left(h_{3 l}\right)$ и динамической связи между $\delta$ - и $\lambda$-интервалами имеем $f(\xi)=\delta_{l-1} \supset \eta^{\prime}$ для любого $\delta$-интервала $\eta \subset \delta_{l-1}$ шага $l$. Стало быть, интервалы $\xi_{s}^{\prime}$ тоже определены и $\xi_{s}^{\prime} \subset \xi_{p}^{\prime}$. Отсюда нетрудно вывести $\left(h_{3(n+1)}\right)$, принимая во внимание соответствующие детали выбора интервала $\lambda_{n+1}$.

$\left(h_{1(n+1)}\right),\left(h_{2(n+1)}\right)$ Заметим, что свойство $\left(h_{1 n}\right)$ (с учетом $\left.\left(h_{2 n}\right)\right)$ влечет соответству ющее свойство продолжаемости для $\delta$-интервалов шага $n$. Примем очередное предположение индукции:

$\left(h_{4 n}\right)$ для любого $\delta$-интервала $\xi$ шага $n$ вьполнены включения $\xi^{\prime} \subset \mathfrak{M}_{n-1}, \xi^{\prime \prime} \subset$ $\mathfrak{M}_{n-2}$ и $\xi^{\prime \prime \prime} \subset \mathfrak{M}_{n-3}$. 
Из этого предположения вытекает, в частности, что для любого $\delta$-интервала $\xi$ шага $n$ такого, что $\xi \subset \delta_{n-1}$, справедливы включения $\xi^{\prime \prime} \subset \delta_{n-2}$ и $\xi^{\prime \prime \prime} \subset \delta_{n-3}$. Действуя как при доказательстве предыдущего свойства, можно показать, что для любого $\lambda$-интервала $\xi$, заполняемого на шаге $n+1$, и для каждого интервала $\xi_{s}$ интервалы $\xi_{s}^{\prime \prime}$ и $\xi_{s}^{\prime \prime \prime}$ определены и

$$
\xi_{s}^{\prime \prime} \subset \xi_{p}^{\prime \prime}=\xi^{\prime}, \quad \xi_{s}^{\prime \prime \prime} \subset \xi_{p}^{\prime \prime \prime}=\xi^{\prime \prime}
$$

Это, в частности, доказьвает свойство $\left(h_{1(n+1)}\right)$ для $\lambda$-интервалов, полученных в результате заполнений.

Что касается включений в $\left(h_{2(n+1)}\right)$, они очевидны для всех $\lambda$-интервалов, полученных в результате заполнений (вследствие $(2.33)$ и свойства $\left(h_{2 n}\right)$ ), а также для всех остальных $\lambda$-интервалов, кроме тех, которые лежат внутри $\varkappa_{n}$. Пусть $\xi$ есть $\lambda$-интервал такой, что $\xi \subset \varkappa_{n}, \xi \cap(S(1), p) \neq \varnothing$. Тогда найдется $\delta$-интервал $\eta \in \mathfrak{m}_{n}$ такой, что $R_{n}(\xi)=\eta$. В силу $\left(h_{4 n}\right)$ имеем $\xi^{\prime \prime \prime}=\left(R_{n}\right)^{-1}\left(\eta^{\prime \prime \prime}\right) \subset$ $\left(R_{n}\right)^{-1}\left(\mathfrak{M}_{n-3}\right)$. Учитывая соответствуюшие детали выбора интервала $\mathfrak{E}_{n+1}$, получаем, что $\xi^{\prime \prime \prime} \subset S(I)$.

Установим свойство $\left(h_{4(n+1)}\right)$. Отметим, что с помощью отображения $S$ (см. свойство $\left.\left(h_{2 n}\right)\right)$ включения (2.33) можно трансформировать в соответствуюшие соотношения для $\delta$-интервалов. Объединяя это с $\left(h_{4 n}\right)$, выводим утверждаемое в $\left(h_{4(n+1)}\right)$ для всех $\delta$-интервалов шага $n+1$, лежащих вне множества $\delta_{n}$. Непосредственньм вычислением с использованием неравенств (2.22), (2.23), (2.30) проверяется, что для любого $\lambda$-интервала $\xi$ такого, что $\xi \subset \lambda_{n} \backslash \lambda_{n+1}$, имеем $\xi_{p}^{\prime \prime \prime} \subset \lambda_{n-1}$. Это позволяет завершить доказательство для остальных $\delta$-интервалов.

Заметим, что отображения $F_{n+1}$ и $G_{n+1}$ зависят непрерьвно от $b$ на интервале $\mathfrak{E}_{n+1}$.

2.5. Оценки мер в фазовом пространстве. В этом пункте устанавливаются свойство (г) отображения $G$ и свойство (v) последовательности множеств $\mathfrak{l}_{n}$, $n \in \mathbb{N} \cup\{0\}$.

Условие суммируемости. Заметим, что для любого $n$ каждая ветвь $U: \Lambda \rightarrow I$ отображений $F_{n}$ или $Z_{n}$ имеет вид $\left.\left.H_{i_{j}} \circ S\right|_{I} \circ \cdots \circ S\right|_{I} \circ H_{i_{1}}$. Обозначим $\mathscr{H}(U)=$ $\mathscr{H}(\Lambda)=j$. Оценки времени возврашаемости индуцированного отображения $G$ основаны на следующей лемме.

Лемма 9. Пусть $U$ есть либо диффеоморфизм глубинь $k, k \in \mathbb{N} \cup\{0\}$, либо одна из ветвей $F_{k}^{t}, t \in \mathbb{N} \cup\{0\}, k \in\{t, t+1, \ldots\}$. Тогда

$$
\mathscr{H}(U)<L 2^{k}
$$

где число $L=L(E)$ введено в п. 2.3.

ДокАЗАТЕЛЬство. Так как для любого диффеоморфизма $H_{0}$ имеем $\mathscr{H}\left(H_{0}\right) \leqslant$ $L-1$, требуемые оценки выполняются на первых шагах конструкции. Предположим, что они проверены при $k \leqslant n, t \leqslant n$. Тогда, к примеру, задавшись одним из диффеоморфизмов $F_{n+1}^{k}, k \leqslant n$, и пользуясь тем, что $F_{n+1}^{k}=U \circ S \circ Q_{l-1}$ для подходящих $l<k, Q_{l}$ и ветви $U=F_{n+1-k+l}^{t}$, можно записать

$$
\mathscr{H}\left(F_{n+1}^{k}\right)=\mathscr{H}\left(U \circ S \circ Q_{l-1}\right)=\mathscr{H}(U)+\mathscr{H}\left(Q_{l-1}\right) \leqslant L 2^{n+1-k+l}+L 2^{l-1} \leqslant L 2^{n+1} .
$$


Действуя аналогично в отношении остальных ветвей, создаваемых на шаге $n+1$, и диффеоморфизмов глубины $n+1$, можно вывести искомые оценки при $k=n+1$, $t \leqslant n+1$ и, тем самьм, доказать лемму по индукции.

Заметим, что любая ветвь $U: \Delta \rightarrow I$ отображения $G$ имеет вид $T_{i_{j}} \circ \cdots \circ T_{i_{1}}$, и полагаем $\mathscr{T}(U)=\mathscr{T}(\Delta)=j$. Каждую из ветвей отображения $G$ вида $F_{n}^{k} \circ S$ будем обозначать через $G_{n}^{k}$. Ясно, что $\mathscr{T}\left(G_{n}^{k}\right)=\mathscr{H}\left(F_{n}^{k}\right)$ и справедливо включение $\Delta G_{n}^{k} \subset \delta_{k-1} \backslash \delta_{k}$. Для доказательства сходимости ряда в условии (г) на отображение $G$ достаточно установить ограниченность последовательности

$$
\mathscr{S}(n)=\sum_{\Delta \in \mathfrak{l}_{n} \backslash \mathfrak{l}_{0}}|\Delta| \mathscr{T}(\Delta), \quad n \in \mathbb{N}
$$

ибо множество $\mathfrak{l}_{0}$ конечно и при $b \neq 2$ отображение $T$ имеет конечное число ветвей. Имеем $\mathscr{S}(n)=\mathscr{S}_{1}(n)+\mathscr{S}_{2}(n)$, где

$$
\mathscr{S}_{1}(n)=\sum_{\Delta \in \mathfrak{l}_{n} \backslash \mathfrak{l}_{0}, \Delta \subset \cup \Delta_{0}}|\Delta| \mathscr{T}(\Delta), \quad \mathscr{S}_{2}(n)=\sum_{k=1}^{n} \sum_{\Delta \in \mathfrak{l}_{n} \backslash \mathfrak{l}_{0}, \Delta \subset \delta_{k-1} \backslash \delta_{k}}|\Delta| \mathscr{T}(\Delta) .
$$

Нетрудно видеть (используя соответствующее свойство для ветвей $F_{k}^{0}$ ), что для любой ветви $G_{k}^{0}$ найдутся определенные диффеоморфизм $T_{0}$ (см. п. 2.3) и ветвь $G_{k-P}^{t}, t \in\left\{\min \left(P_{2}+1, k-P\right), \ldots, k-P\right\}$, такие, что $G_{k}^{0}=G_{k-P}^{t} \circ T_{0}$. Учитывая это, можно показать, что $\mathscr{S}_{1}(n)<$ const $\cdot \mathscr{S}_{2}(n-P)$, где константа зависит только от параметров предварительной конструкции. Стало быть, достаточно рассмотреть последовательность $\mathscr{S}_{2}(n), n \in \mathbb{N}$. Запишем

$$
\mathscr{S}_{2}(n)=\sum_{k=1}^{n} \sum_{j=k}^{n} \sum_{G_{j}^{k}}\left|\Delta G_{j}^{k}\right| \mathscr{T}\left(G_{j}^{k}\right)
$$

Одним из свойств конструкции, изложенной в п. 2.4, является то, что ветви отображения $G$, построенные на шаге $j, j \geqslant 1$, определены внутри множества $\mathfrak{M}_{j-1}$. Поэтому существенной для дальнейшего является следующая лемма.

ЛЕмма 10. Для любого $k \geqslant 1$ и для любого $j \geqslant k$

$$
\left|\bigcup_{\xi \in \mathfrak{m}_{j}, \xi \subset \delta_{k-1} \backslash \delta_{k}} \xi\right|<2 \sqrt{\lambda}\left(4 \tau_{2} \sqrt{\lambda}\right)^{j-k}\left|\delta_{k-1} \backslash \delta_{k}\right|
$$

ДоказАТЕльство. Неравенства $\left(2.35_{k k}\right), k \geqslant 1$, доказываются по индукции в том же духе, как это делается в [2; лемма 2], с учетом соотношений $(2.14),(2.30)$ и свойства $\left(h_{3 n}\right)$. В качестве пояснения добавим лишь, что, во-первых, если $\xi$ есть $\lambda$ - или $\delta$-интервал и $f: \xi \rightarrow \delta_{l}$ есть соответствующий диффеоморфизм, из неравенств $\left(2.35_{(l+1)(l+1)}\right)$ и $(2.23)$ вытекает, что

$$
\left|\xi_{p}\right|+\sum\left|\xi_{s}\right|<4 \tau_{2} \sqrt{\lambda}|\xi|
$$

Во-вторых, предположим, что $\xi \subset \lambda_{n}$. Легко показать, опираясь на свойства $\left(b_{5 k}\right)$, $k<n$, что диффеоморфизмы и $\lambda$-интервалы, построенные внутри $\xi^{\prime} \backslash \xi$, получаются 
заполнением интервала $\xi^{\prime}$, в частности находятся в биективном соответствии с ветвями отображения $G_{l}$, определенными внутри множества $\delta_{l-1} \backslash \delta_{l}$, и $\delta$-интервалами шага $l$. Поэтому суммарную меру $\lambda$-интервалов в $\xi^{\prime} \backslash \xi$ также можно оценивать, исходя из неравенства $\left(2.35_{l l}\right)$.

Учитывая сказанное, неравенства $\left(2.35_{k j}\right)$ доказываются индукцией по $j$ при фиксированном $k$.

С помощью лемм 9,10, неравенств $(2.14),(2.22)$ и выражения $(2.34)$ оценим

$$
\begin{aligned}
\mathscr{S}_{2}(n) & <\sum_{k=1}^{n}\left(L 2^{k}\left|\delta_{k-1} \backslash \delta_{k}\right|+\sum_{j=k+1}^{n} L 2^{j} 2 \sqrt{\lambda}\left(4 \tau_{2} \sqrt{\lambda}\right)^{j-1-k}\left|\delta_{k-1} \backslash \delta_{k}\right|\right) \\
& <L \sum_{k=1}^{n} 2^{k}\left|\delta_{k-1}\right|\left(1+\sum_{j=1}^{n-k} 2^{j} 2 \sqrt{\lambda}\left(4 \tau_{2} \sqrt{\lambda}\right)^{j-1}\right) \\
& <\operatorname{const} \sum_{k=1}^{n} 2^{k} \sqrt{2 \alpha \lambda^{k}}(1+\varepsilon)<\text { const }
\end{aligned}
$$

принимая во внимание выбор $\lambda$ (условие (2.30)).

Асимптотика мер множеств $\mathfrak{M}_{n}$. Из неравенства (2.36) с учетом условия (2.30) вьводится следующий результат.

ЛЕмма 11. Для любого п справедлива оценка

$$
\left|\mathfrak{M}_{n}\right|<10^{-n}\left|\mathfrak{M}_{0}\right|
$$

2.6. Оценки меры в пространстве параметра $b$. Напомним, что на шаге $n+1$ зафиксирован составляющий интервал $\mathfrak{E}_{n}$ множества $\mathfrak{D}_{n}$ такой, что при изменении параметра $b$ в пределах $\mathfrak{E}_{n}$ критическое значение $S(1)$ с почти единичной скоростью пробегает интервал $\varkappa_{n}$ глубины $n$. При этом часть множества $\mathfrak{D}_{n+1}$ в интервале $\mathfrak{E}_{n}$ состоит из тех $b$, при которых точка $S(1)$ лежит внутри относительно неподвижных интервалов глубины $n+1$, в совокупности составляющих семейство $\mathfrak{p}_{n+1}$. Стало быть, мы находимся в рамках ситуации, описываемой следующей леммой.

Лемма 12. Рассмотрим дифференцируемые действительные функиии $\zeta$, $\phi_{i}, \psi_{i}, i=1, \ldots, j$, такие, что для некоторых малых положсительных чисел $\varepsilon_{1}, \varepsilon_{2}$ и $\varepsilon_{3}$ справедливы неравенства

$$
\begin{gathered}
\phi_{1}<\psi_{1} \leqslant \phi_{2}<\psi_{2} \leqslant \cdots \leqslant \phi_{j}<\psi_{j}, \quad 1-\varepsilon_{1}<\left|\zeta^{\prime}\right|<1+\varepsilon_{1}, \\
\max _{i=1, \ldots, j} \max \left(\left|\phi_{i}^{\prime}\right|,\left|\psi_{i}^{\prime}\right|\right)<\varepsilon_{2}, \quad \max _{i=1, \ldots, j} \frac{\left|\left(\psi_{i}-\phi_{i}\right)^{\prime}\right|}{\left|\psi_{i}-\phi_{i}\right|}<\varepsilon_{3} .
\end{gathered}
$$

Определим числа $x_{i}^{\prime}, x_{i}^{\prime \prime}, i=1, \ldots, j$, с помощъю уравнений $\zeta\left(x_{i}^{\prime}\right)=\phi_{i}\left(x_{i}^{\prime}\right)$, $\zeta\left(x_{i}^{\prime \prime}\right)=\psi_{i}\left(x_{i}^{\prime \prime}\right)$. Тогда для любого $x \in\left[x_{1}^{\prime}, x_{j}^{\prime \prime}\right]$ имеем

$$
\sum_{i=1}^{j}\left|x_{i}^{\prime \prime}-x_{i}^{\prime}\right| \leqslant \frac{\sum_{i=1}^{j}\left|\psi_{i}(x)-\phi_{i}(x)\right|}{\left(1-\varepsilon_{1}-\varepsilon_{2}\right)\left(1-\varepsilon_{3}\left|x_{j}^{\prime \prime}-x_{1}^{\prime}\right|\right)}
$$

при условии, что $\varepsilon_{3}\left|x_{j}^{\prime \prime}-x_{1}^{\prime}\right|<1$. 
ЗАмЕЧАНИЕ 10. Эта лемма неявно, хотя и в ином ключе, использовалась в $[2 ;$ c. 69$]$.

ДокАЗАТЕЛЬСТво ЛЕммы 12. Нетрудно показать, что в условиях леммы для каждого $i$ и для любых $x \in\left[x_{1}^{\prime}, x_{j}^{\prime \prime}\right], y \in\left[x_{1}^{\prime}, x_{j}^{\prime \prime}\right]$

$$
\frac{\left|\psi_{i}(x)-\phi_{i}(x)\right|}{\left|\psi_{i}(y)-\phi_{i}(y)\right|} \leqslant \frac{1}{1-\varepsilon_{3}\left|x_{j}^{\prime \prime}-x_{1}^{\prime}\right|} .
$$

Опираясь на эти оценки и учитывая определение точек $x_{i}^{\prime}, x_{i}^{\prime \prime}$, путем несложных преобразований получаем

$$
\begin{aligned}
& \sum_{i=1}^{j}\left|x_{i}^{\prime \prime}-x_{i}^{\prime}\right| \leqslant\left(1-\varepsilon_{1}\right)^{-1}\left(\sum_{i=1}^{j}\left|\psi_{i}\left(x_{i}^{\prime \prime}\right)-\psi_{i}\left(x_{i}^{\prime}\right)\right|+\sum_{i=1}^{j}\left|\psi_{i}\left(x_{i}^{\prime}\right)-\phi_{i}\left(x_{i}^{\prime}\right)\right|\right) \\
& \quad \leqslant\left(1-\varepsilon_{1}\right)^{-1}\left(\varepsilon_{2} \sum_{i=1}^{j}\left|x_{i}^{\prime \prime}-x_{i}^{\prime}\right|+\frac{1}{1-\varepsilon_{3}\left|x_{j}^{\prime \prime}-x_{1}^{\prime}\right|} \sum_{i=1}^{j}\left|\psi_{i}(x)-\phi_{i}(x)\right|\right)
\end{aligned}
$$

для любого $x \in\left[x_{1}^{\prime}, x_{j}^{\prime \prime}\right]$. Разрешая полученное неравенство относительно числа $\sum_{i=1}^{j}\left|x_{i}^{\prime \prime}-x_{i}^{\prime}\right|$, завершаем доказательство.

Чтобы задействовать лемму 12 при оценивании $\left|\mathfrak{E}_{n} \backslash \mathfrak{D}_{n+1}\right|$, надо уметь контролировать скорости $\lambda$-интервалов. Для интервала $\xi=\left(y^{\prime}, y^{\prime \prime}\right)$, зависящего от параметра $b$, обозначим

$$
\mathscr{V}(\xi)=\max \left(\left|\partial_{b} y^{\prime}\right|,\left|\partial_{b} y^{\prime \prime}\right|\right), \quad \mathscr{R}(\xi)=\frac{\left|\partial_{b}\right| \xi||}{|\xi|} .
$$

Скорости $\mathscr{V}$ описываются леммой 8 с учетом того, что скорости точек $p$ и $p^{-1}$ пренебрежимо малы. Оценивание приведенных скоростей $\mathscr{R}$ осуществляется индуктивно (лемма 15). При этом оказывается нужным знание того, как функция $\lambda$-интервала $\mathscr{R}(\cdot)$ ведет себя относительно преобразований этих интервалов. Пусть интервал $\xi$ отображается на другой интервал $\eta$ с помощњю диффеоморфизма $F$, зависящего от параметра $b$. Тогда нетрудно проверить, что

$$
\mathscr{R}(\xi)<\mathscr{R}(F)+\tau(F, \xi) \mathscr{R}(\eta)+\tau(F, \xi) \mu(F, \xi) \mathscr{V}(\eta),
$$

где число

$$
\mathscr{R}(F)=\sup _{x \in \eta}\left|\frac{\partial_{b} \partial F^{-1}(b, x)}{\partial F^{-1}(b, x)}\right|,
$$

вообше говоря, характеризует приведенную скорость интервала определения $F$ (по поводу обозначения $\mu(F, \xi)$ см. п. 2.1). Совместное использование одного символа $\mathscr{R}$ для обозначения разных величин целесообразно, ибо эти величины, по сути дела, выражают одно и то же. То же самое справедливо и в отношении символа $\mathscr{V}$.

Сказанное является преамбулой к следующей формулировке. Положим

$$
\begin{gathered}
D_{1}=\sup _{x \in I}\left|\partial^{2} S(x)\right|>1, \quad D_{2}=\sup _{H_{0}} \mathscr{R}\left(H_{0}\right), \quad D_{3}=D_{2}+C_{5}, \\
D_{4}=B_{1}\left(\frac{D_{1} B_{4}^{2}}{(2(1-\varepsilon))^{2}}+\frac{\mu B_{4}}{2(1-\varepsilon)}\right)
\end{gathered}
$$

где $C_{5}$ есть константа из леммы $5, \mu=\mu(I, \widehat{I})$ (см. замечание 6$)$. 
Лемма 13. Для любых чисел $k \in \mathbb{N} \cup\{0\}, t=1, \ldots, k$ справедливы следующие оценки:

$$
\begin{gathered}
\max \left(\sup _{Q_{k}} \mathscr{R}\left(Q_{k}\right), \sup _{F_{k}^{t}} \mathscr{R}\left(F_{k}^{t}\right)\right)<D_{3} 2^{k}+2 D_{4} \sum_{i=0}^{k-1} 2^{k-1-i}\left(\frac{1}{\alpha \lambda^{i}}+B_{3}\right)+2 \varepsilon \sum_{i=0}^{k-1} 2^{i} \\
\sup _{F_{k}^{0}} \mathscr{R}\left(F_{k}^{0}\right)<D_{3} 2^{k}+D_{4} \sum_{i=0}^{k} 2^{k-i}\left(\frac{1}{\alpha \lambda^{i}}+B_{3}\right)+\varepsilon \sum_{i=0}^{k} 2^{i} .
\end{gathered}
$$

ДоКАЗАТЕЛЬСтво. Предположим, что оценки леммы верны для $k \leqslant n$, и установим их для $k=n+1$. Рассмотрим композицию $W=V \circ S \circ U$, где $U$ и $V$ суть диффеоморфизмы на $I$, определенные внутри интервала $(0, p)$, и предположим, что $\Lambda V \subset S(I)$. Используя формулы (2.9), (2.8) и (2.7) (чтобы посчитать $\left.\partial_{b}\left((V \circ S)^{-1}\right)(x)\right)$, запишем

$$
\begin{aligned}
\frac{\partial_{b} \partial W^{-1}(x)}{\partial W^{-1}(x)}= & \frac{\partial_{b} \partial U^{-1}(U(y))}{\partial U^{-1}(U(y))}+\frac{\partial^{2} U}{\partial U^{2}}\left(\frac{\partial_{b} V}{\partial V} \frac{1}{\partial S}+\frac{\partial_{b} S}{\partial S}\right) \\
& +\frac{\partial^{2} S}{\partial S^{2}} \partial_{b} S-\frac{\partial_{b} \partial S}{\partial S}+\frac{\partial^{2} S}{\partial S^{2}} \frac{\partial_{b} V}{\partial V}+\frac{\partial_{b} \partial V^{-1}(x)}{\partial V^{-1}(x)}
\end{aligned}
$$

где $x=W(y)$ и аргументами $U, S$ и $V$ являются $y, U(y)$ и $S \circ U(y)$ соответственно. Таким образом, если диффеоморфизм $U$ продолжаемьй, то, учитывая замечание 8 , имеем

$$
\mathscr{R}(W) \leqslant \mathscr{R}(U)+\mathscr{R}(V)+(\mathscr{V}(V)+1+\varepsilon)\left(D_{1} \sup \frac{1}{\partial S^{2}}+\mu \sup \frac{1}{|\partial S|}\right)+\varepsilon
$$

Нетрудно видеть, что третье слагаемое в правой части (2.39), которое обозначим через $\mathscr{C}(V)$, зависит только от диффеоморфизма $V$. Предположим, что $V=F_{j}^{i}$, $i \geqslant 1$, и оценим

$$
\begin{aligned}
\mathscr{C}(V) & <\frac{B_{1}}{\sqrt{\lambda^{j-i}}}\left(\frac{D_{1}}{(2(1-\varepsilon))^{2} \alpha \lambda^{i}}+\frac{\mu}{2(1-\varepsilon) \sqrt{\alpha \lambda^{i}}}\right) \\
& <\frac{1}{\alpha \lambda^{j}} D_{4}<\left(\frac{1}{\alpha \lambda^{j}}+B_{3}\right) D_{4}
\end{aligned}
$$

учитывая оценки леммы 8 , неравенство $i \leqslant j$ и тот факт, что число $\alpha \lambda^{i}$, вообще говоря, очень мало. Аналогично, если $V=F_{j}^{0}$, то

$$
\mathscr{C}(V)<\left(\frac{1}{\sqrt{\alpha \lambda^{j}}}+B_{3}\right)\left(\frac{D_{1} B_{4}^{2}}{(2(1-\varepsilon))^{2}}+\frac{\mu B_{4}}{2(1-\varepsilon)}\right)<\left(\frac{1}{\alpha \lambda^{j}}+B_{3}\right) D_{4} .
$$

Зафиксируем диффеоморфизм $Q_{n+1}$ и вспомним, что $Q_{n+1}=F \circ S \circ Q_{n}$ для определенного диффеоморфизма $Q_{n}$ и подходящей ветви $F$ отображения $F_{n}$. Заметим, что для любого фиксированного $t \in\{0,1, \ldots, n\}$ мажоранта для $\mathscr{R}\left(F_{k}^{t}\right)$ в 
формулировке леммы является наибольшей при $k=n$. Поэтому предположим, что $F=F_{n}^{0}$, и, используя соотношения (2.39) и (2.41), запишем

$$
\begin{aligned}
\mathscr{R}\left(Q_{n+1}\right)< & D_{3} 2^{n}+2 D_{4} \sum_{i=0}^{n-1} 2^{n-1-i}\left(\frac{1}{\alpha \lambda^{i}}+B_{3}\right)+2 \varepsilon \sum_{i=0}^{n-1} 2^{i} \\
& +D_{3} 2^{n}+D_{4} \sum_{i=0}^{n} 2^{n-i}\left(\frac{1}{\alpha \lambda^{i}}+B_{3}\right)+\varepsilon \sum_{i=0}^{n} 2^{i}+\left(\frac{1}{\alpha \lambda^{n}}+B_{3}\right) D_{4}+\varepsilon \\
= & D_{3} 2^{n+1}+D_{4}\left(\sum_{i=0}^{n-1} 2^{n-i}\left(\frac{1}{\alpha \lambda^{i}}+B_{3}\right)+\left(\frac{1}{\alpha \lambda^{n}}+B_{3}\right)\right. \\
& \left.+\sum_{i=0}^{n} 2^{n-i}\left(\frac{1}{\alpha \lambda^{i}}+B_{3}\right)\right)+\varepsilon\left(\sum_{i=1}^{n} 2^{i}+1+\sum_{i=0}^{n} 2^{i}\right)
\end{aligned}
$$

что и требовалось. Оценка в случае, когда $F=F_{n}^{t}, t \in\{1, \ldots, n\}$, аналогична и основывается на неравенстве (2.40).

Оценки леммы для ветвей $F_{n+1}^{t}, t=0,1, \ldots, n+1$, проводятся теми же самыми средствами.

Следующая лемма используется в доказательстве леммы 15.

ЛЕмма 14. Для любого $n \geqslant-(P+3)$ имеем

$$
\mathscr{R}\left(\delta_{n}\right)<\frac{1}{(1-\varepsilon)^{2} \alpha \lambda^{n}}, \quad \mathscr{V}\left(\delta_{n}\right)<\frac{1}{(1-\varepsilon) \sqrt{\alpha \lambda^{n}}} .
$$

Этот вычислительный факт является прямьм следствием замечания 8, неравенств (2.14), (2.22) и леммы 8.

Перед форомулировкой следуюшей леммы договоримся обозначать символом $\xi_{n}^{k}$ (или $\eta_{n}^{k}$ ) каждый из $\lambda$-интервалов, фигурирующий в конструкции после шага $n$ и расположенный внутри множества $\lambda_{k-1} \backslash \lambda_{k}, k=P_{2}+1, \ldots, n$. Рассмотрим один из $\lambda$-интервалов $\xi$. Вследствие свойства $\left(h_{1 n}\right)$ при подходящем $n$ интервалы $\xi$ и $\xi^{\prime}$ находятся в поле действия принципа Кёбе с константой, скажем, $\tau_{3}$. Предположим, что параметры $P$ и $P_{1}$ удовлетворяют условиям

$$
\tau_{3} 2^{1-P}<1, \quad \tau_{3} 2^{1-(1-\omega) P_{1}}<1
$$

увеличив их в случае надобности. Положим

$$
D_{5}=4 D_{4} \tau_{3}>D_{4}
$$

и обратимся к доказательству следующей леммы.

ЛЕмма 15. Предположим, что $\xi$ есть $\lambda$-интервал после шага $k$. Тогда

$$
\mathscr{R}(\xi)<D_{3} 2^{k}+D_{5} \sum_{i=0}^{k} 2^{k-i}\left(\frac{1}{\alpha \lambda^{i}}+B_{3}\right)+\varepsilon \sum_{i=0}^{k} 2^{i}
$$


ДокаЗАТЕльство. Допустим, что утверждаемое в лемме доказано для $k \leqslant n$, и выведем требуемую оценку при $k=n+1$. Рассмотрим $\lambda$-интервал $\xi$. Для него существуют четыре возможности. Именно найдутся диффеоморфизм $F=F_{\xi}$ и число $j$ такие, что либо $F(\xi)=\delta_{j}$, либо $(S \circ F)(\xi)=\eta$ для некоторого интервала $\eta=\eta_{j}^{t}, t \in\left\{0, P_{2}+1, \ldots, j\right\}$. Если $\xi$ есть один из интервалов $\xi_{n+1}^{0}$, то $F=H_{0}$, $j=n+1-P, t>0$. В противном случае, когда $\xi=\xi_{n+1}^{m}, m \in\left\{P_{2}+1, \ldots, n+1\right\}$, имеем $F=Q_{l-1}, j=n+1-m+l$, где $l \leqslant m-1$. Фигурирующий здесь образ интервала $\xi$ будем обозначать через $\eta_{\xi}$.

Предположим, что некоторая композиция $S \circ F$, где $F$ есть продолжаемый дифифеоморфизм, отображает $\lambda$-интервал $\xi_{1}$ на $\lambda$-интервал $\xi_{2}$. Используя формулу

$$
\frac{\partial^{2}(S \circ F)}{\partial(S \circ F)^{2}}=\frac{\partial^{2} S}{\partial S^{2}}+\frac{1}{\partial S} \frac{\partial^{2} F}{\partial F^{2}},
$$

оценку (2.37), выражения (2.9), (2.8) и замечание 8 , получаем

$$
\begin{aligned}
\mathscr{R}\left(\xi_{1}\right) \leqslant & \mathscr{R}(F)+\tau_{3} \mathscr{R}\left(\xi_{2}\right) \\
& +\left(\tau_{3} \mathscr{V}\left(\xi_{2}\right)+1+\varepsilon\right)\left(D_{1} \sup \frac{1}{\partial S^{2}}+\mu \sup \frac{1}{|\partial S|}\right)+\varepsilon .
\end{aligned}
$$

Нетрудно видеть, что третье слагаемое в правой части (2.44), которое обозначим через $\mathscr{C}\left(\xi_{2}\right)$, зависит только от $\xi_{2}$. Заметим, что для любых $t, j, t \leqslant j$, граница любого из интервалов $\xi_{j}^{t}$ состоит из граничных точек прилегающих интервалов $\Lambda F_{j}^{t}$. Это означает, что к оцениванию $\mathscr{V}\left(\xi_{j}^{t}\right)$ применимы соответствующие неравенства леммы $8(j)$. Учитьвая это замечание и действуя как в доказательстве леммы 13 , можно показать, что

$$
\sup _{\xi_{j}^{t}} \mathscr{C}\left(\xi_{j}^{t}\right)<\left(\frac{1}{\alpha \lambda^{j}}+B_{3}\right) D_{5} .
$$

Предположим, что $\xi=\xi_{n+1}^{m}, \eta_{\xi}=\eta=\eta_{n+1-m+l}^{t}, F_{\xi}=Q_{l-1}$. Используя соотношения (2.44) и (2.45) с $\xi_{1}=\xi, \xi_{2}=\eta$ и $F \stackrel{m}{=} Q_{l-1}$, лемму 13 , а также предположение индукции, оценим

$$
\begin{aligned}
\mathscr{R}(\xi) \leqslant & D_{3} 2^{l-1}+2 D_{4} \sum_{i=0}^{l-2} 2^{l-2-i}\left(\frac{1}{\alpha \lambda^{i}}+B_{3}\right)+2 \varepsilon \sum_{i=0}^{l-2} 2^{i} \\
& +\tau_{3} D_{3} 2^{n+1-m+l}+\tau_{3} D_{5} \sum_{i=0}^{n+1-m+l} 2^{n+1-m+l-i}\left(\frac{1}{\alpha \lambda^{i}}+B_{3}\right) \\
& +\tau_{3} \varepsilon \sum_{i=0}^{n+1-m+l} 2^{i}+\left(\frac{1}{\alpha \lambda^{n+1-m+l}}+B_{3}\right) D_{5}+\varepsilon=\mathscr{W}
\end{aligned}
$$

Ввиду второго из условий в $(2.42)$ и свойства $\left(b_{6}\right)$ заметим, что $\tau_{3} 2^{1-m+l} \leqslant 1$. Отсюда

$$
\begin{aligned}
\mathscr{W} \leqslant & D_{3}\left(2^{l-1}+\tau_{3} 2^{1-m+l} 2^{n}\right)+D_{5}\left(\sum_{i=0}^{n-2} 2^{n-1-i}\left(\frac{1}{\alpha \lambda^{i}}+B_{3}\right)\right. \\
& \left.+\left(\frac{1}{\alpha \lambda^{n}}+B_{3}\right)+\tau_{3} 2^{1-m+l} \sum_{i=0}^{n} 2^{n-i}\left(\frac{1}{\alpha \lambda^{i}}+B_{3}\right)\right) \\
& +\varepsilon\left(\sum_{i=1}^{n-1} 2^{i}+1+\tau_{3} 2^{1-m+l} \sum_{i=m-l-1}^{n} 2^{i}\right)
\end{aligned}
$$


что приводит к требуемой оценке.

Случай, когда $\xi=\xi_{n+1}^{0}, \eta_{\xi}=\eta_{n+1-P}^{t}, F_{\xi}=H_{0}$, рассматривается в той же самой манере с учетом первого из условий в (2.42). Если же $\eta_{\xi}=\delta_{j}$, то вывод искомых соотношений упрощается и сводится к непосредственному применению неравенства (2.37) и леммы 14.

Вспомним, что множество $\mathfrak{E}_{n} \backslash \mathfrak{D}_{n+1}$ состоит $(\bmod 0)$ из тех значений параметра $b$, при которых $S(1) \in\left(R_{n}\right)^{-1}\left(\mathfrak{M}_{n-3}\right)$. Соединяя леммы $13-15$ и оценку $(2.44)$, получаем

$$
\sup _{b \in \mathfrak{E}_{n}} \sup _{\xi \in \mathfrak{m}_{n-3}} \mathscr{R}\left(\left(R_{n}\right)^{-1}(\xi)\right) \leqslant D_{6} \lambda^{-n}
$$

где константа $D_{6}$ зависит от чисел $\varepsilon, \tau_{3}, B_{3}, D_{3}, D_{4}, D_{5}, \alpha$. Дополнительно к условиям (2.17)-(2.19) предположим, что для любого $n \in \mathbb{N} \cup\{0\}$

$$
\beta \varkappa^{n} D_{6} \lambda^{-n}<\frac{1}{10} \text {. }
$$

Отметим, что вследствие замечания 8 , леммы $8(n)$ и неравенства $(2.17)$ выполнены посылки леммы 6 с $\varepsilon_{1}=\varepsilon_{2}=10^{-1}$, согласно которой справедливо неравенство

$$
\frac{5}{6}\left|\varkappa_{n}(b)\right| \leqslant\left|\mathfrak{E}_{n}\right| \leqslant \frac{5}{4}\left|\varkappa_{n}(b)\right| \leqslant \frac{5}{4} \beta \varkappa^{n}
$$

для любой из граничных точек $b$ интервала $\mathfrak{E}_{n}$.

Принимая во внимание соотношения (2.46) и (2.47), оценку меры множества $\mathfrak{E}_{n} \backslash \mathfrak{D}_{n+1}$ можно провести исходя из леммы 12 с $\varepsilon_{1}=\varepsilon_{2}=10^{-1}$ при условии, что $\varepsilon_{3}\left|x_{j}^{\prime \prime}-x_{1}^{\prime}\right| \leqslant 8^{-1}$. В результате получаем, что для любого $n$ и для любого составляющего интервала $\mathfrak{E}_{n}=\left(b_{n}^{\prime}, b_{n}^{\prime \prime}\right)$ множества $\mathfrak{D}_{n}$

$$
\begin{aligned}
\frac{\left|\mathfrak{E}_{n} \backslash \mathfrak{D}_{n+1}\right|}{\left|\mathfrak{E}_{n}\right|} & \leqslant \frac{10}{7} \frac{\left|\left(R_{n}\right)^{-1}\left(\mathfrak{M}_{n-3}\right)\left(b_{n}^{\prime}\right)\right|}{\left|\mathfrak{E}_{n}\right|} \leqslant \frac{10}{7} \frac{\tau\left|\mathfrak{M}_{n-3}\left(b_{n}^{\prime}\right)\right|\left|\varkappa_{n}\left(b_{n}^{\prime}\right)\right|}{|I|\left|\mathfrak{E}_{n}\right|} \\
& \leqslant \frac{10 \cdot 6}{7 \cdot 5} \frac{\tau 10^{-n+3}\left|\mathfrak{M}_{0}\right|\left|\mathfrak{E}_{n}\right|}{|I|\left|\mathfrak{E}_{n}\right|} \leqslant D_{7} 10^{-n}\left|\mathfrak{M}_{0}\right|
\end{aligned}
$$

(см. лемму 11).

Как показано в работе [9], $\left|\mathfrak{M}_{0}\right| \leqslant$ const $\left|\delta_{0}\right|$ с константой, зависящей от свойств растяжения отображения $T$ при $b=2$. С учетом оценок $(2.14)$ и (2.22) это влечет, что $\left|\mathfrak{M}_{0}\right| \leqslant \operatorname{const} \sqrt{\alpha}$. Сделанное замечание и неравенство (2.48) говорят об экспоненциальном убывании мер множеств $\mathfrak{D}_{n} \backslash \mathfrak{D}_{n+1}$ (свойство (viii) последовательности $\mathfrak{D}_{n}, n \in \mathbb{N} \cup\{0\}$ ) и влекут сушествование константы $D_{8}$, не зависяшей от числа $\alpha$ и такой, что для любого интервала $\mathfrak{E}_{0}$

$$
\frac{\left|\mathfrak{G}(\alpha) \cap \mathfrak{E}_{0}\right|}{\left|\mathfrak{E}_{0}\right|} \geqslant 1-D_{8} \sqrt{\alpha} .
$$

С помощью лемм 4-6 неравенства в п. (iii) леммы 3 трансформируются в оценки мер интервалов $\mathfrak{E}_{0}$. Благодаря последним нижняя плотность множества $\mathfrak{G}(\alpha)$ в точке $b=2$ может быть сделана сколь угодно близкой к единице за счет выбора $\alpha$. Тем самым, доказано утверждение о точке плотности в теореме 3. 
2.7. Построение множества $\mathfrak{S}_{1}$. Зафиксируем $\alpha$. Отметим, что для каждого $n$ множество $\mathfrak{D}_{n}$ зависит от $c$. Будем называть әлементарными непустые множества вида

$$
\left\{(c, b) \in \mathbb{R}^{2}: c^{\prime} \leqslant c<c^{\prime \prime}, b \in \mathfrak{E}_{n}(c)\right\},
$$

где для любого $c$ множество $\mathfrak{E}_{n}(c)$ является составляющим интервалом множества $\mathfrak{D}_{n}(c)$, гладко зависящим от $c, n \in \mathbb{N} \cup\{0\}$. Если $\mathfrak{A}$ есть элементарное множество вида (2.49), то любое из множеств $\{c\} \times \mathfrak{E}_{n}(c)$ называется слоем множества $\mathfrak{A}$, в то время как полуинтервал $\left[c^{\prime}, c^{\prime \prime}\right)$ называется проекиией множества $\mathfrak{A}$ и обозначается через $\mathscr{P}(\mathfrak{A})$. Для произвольного $n \in \mathbb{N} \cup\{0\}$ и любой пары $(c, b)$ такой, что $c_{3} \leqslant c, b \in \mathfrak{D}_{n}(c)$, обозначим через $\mathfrak{B}_{n, c, b}$ множество $\{c\} \times \mathfrak{E}_{n}$, где $\mathfrak{E}_{n}$ есть составляющий интервал множества $\mathfrak{D}_{n}(c)$, содержащий $b$.

Множество

$$
\mathfrak{S}_{1}=\mathfrak{S}_{1}(\alpha)=\bigcup_{c \geqslant c_{3}}\left\{(a, b) \in \mathbb{R}^{2}: b \in \mathfrak{G}_{c}(\alpha), a=c(2-b)^{-3}\right\}
$$

измеримо как счетное пересечение множеств

$$
\bigcup_{c \geqslant c_{3}}\left\{(a, b) \in \mathbb{R}^{2}: b \in \mathfrak{D}_{n}(c), a=c(2-b)^{-3}\right\}
$$

с учетом следующего факта.

Лемма 16. Для любъх $n \in \mathbb{N} \cup\{0\}, c \in\left[c_{3}, \infty\right) u b \in \mathfrak{D}_{n}(c)$ cyществует әлементарное множество $\mathfrak{A}$ такое, что множсество $\mathfrak{B}_{n, c, b}$ является одним из слоев $\mathfrak{A}$.

Действительно, тогда каждое из множеств $\left\{(c, b) \in \mathbb{R}^{2}: c_{3} \leqslant c, b \in \mathfrak{D}_{n}(c)\right\}$ измеримо как не более чем счетное объединение классов эквивалентности по следующему отношению. Две пары $\left(c^{\prime}, b^{\prime}\right)$ и $\left(c^{\prime \prime}, b^{\prime \prime}\right)$ считаются эквивалентными, если сушествует элементарное множество $\mathfrak{A}$ такое, что множества $\mathfrak{B}_{n, c^{\prime}, b^{\prime}}$ и $\mathfrak{B}_{n, c^{\prime \prime}, b^{\prime \prime}}$ являются слоями $\mathfrak{A}$ (разумеется, это отношение зависит от $n$ ).

ДоКАЗАТЕЛьство лЕммы 16. Сперва отметим, что любая итерация отображения $S$ представляет собой рациональную функцию переменных $c, b$ и $x$. Поэтому у является алгебраической функцией переменных $c, b$, коль скоро $y$ есть граничная точка одного из интервалов множеств $\mathfrak{k}_{n}$ или $\mathfrak{n}_{n}$. Аналогично, если в этих условиях при фиксированном $c$ число $b$ определяется уравнением $S(1)=y$, то $b$ связано алгебраической зависимостью с $c$.

Ясно, что лемма выполняется при $n=0$, причем в качестве элементарных множеств, фигурирующих в ее формулировке, можно взять множества вида $\{(c, b) \in$ $\left.\mathbb{R}^{2}: c_{3} \leqslant c, b \in \mathfrak{E}_{0}(c)\right\}$. Если $\mathfrak{A}$ есть одно из таких множеств, то все ветви отображения $\bar{H}$ меняются непрерывно в зависимости от $(c, b) \in \mathfrak{A}$. Доказательство леммы проведем с помощью индукции по $n$. При этом будем опираться на то, что для любого $n \in \mathbb{N} \cup\{0\}$ интервалы фазового пространства, введенные в рассмотрение к шагу $n+1$, в определенном смысле регулярно зависят от $c, b$. Это означает, что в формулировке леммы можно дополнительно считать, что

$\left(b_{7 n}\right)$ каждый интервал множества $\mathfrak{k}_{n}$ или $\mathfrak{n}_{n}$ гладко зависит от $(c, b) \in \mathfrak{A}$;

$\left(h_{5 n}\right)$ интервал $\lambda_{n}$, а также каждый из $\lambda$-интервалов $\xi$ вместе со своим расширением $\xi^{\prime}$ зависят гладко от $(c, b) \in \mathfrak{A}$. 
С этого момента зададимся числом $c, c_{3} \leqslant c$. В дополнение к сказанному в п. 2.4 уточним некоторые детали выбора интервалов $\lambda_{i}, i=-P-3, \ldots,-1,0$. Зафиксируем составляющий интервал $\mathfrak{E}_{0}=\left(b_{0}^{\prime}(c), b_{0}^{\prime \prime}(c)\right)$ и число $i \in\{-P-3, \ldots,-1,0\}$, положим $b=b_{0}^{\prime \prime}(c)$ и допустим, что $\frac{3}{2} \alpha \lambda^{i}=y_{m}$ для некоторого $m, m>m_{0}$. По соображениям алгебраичности сушествует элементарное множество $\mathfrak{A}$ со слоем $\{c\} \times \mathfrak{E}_{0}$ такое, что имеем альтернативу: либо $\frac{3}{2} \alpha \lambda^{i}=y_{m}$ для любого $c^{\prime} \in \mathscr{P}(\mathfrak{A})$, либо для любого $c^{\prime} \in \mathscr{P}(\mathfrak{A}), c \neq c^{\prime}$, имеем $\frac{3}{2} \alpha \lambda^{i} \neq y_{m}$ (здесь имеется в виду, что $\left.b=b_{0}^{\prime \prime}\left(c^{\prime}\right)\right)$. В первом случае $n_{i}=m$, во втором случае полагаем $n_{i}=m$, если $\frac{3}{2} \alpha \lambda^{i}<y_{m}, c^{\prime}>c$, и, напротив, $n_{i}=m-1$, если $\frac{3}{2} \alpha \lambda^{i}>y_{m}, c^{\prime}>c$. Нетрудно убедиться, что после сделанных оговорок вьполнены свойства $\left(b_{7 n}\right)$ и $\left(h_{5 n}\right)$ при $n=0$.

Зафиксируем $n \in \mathbb{N} \cup\{0\}$ и предположим, что лемма и свойства $\left(b_{7 k}\right)$ и $\left(h_{5 k}\right)$ установлены для $k \leqslant n$. Зададимся числом $b, b \in \mathfrak{D}_{n+1}(c)$, и пусть $\mathfrak{E}_{n}$ есть составляющий интервал множества $\mathfrak{D}_{n}(c)$, содержащий $b$. Согласно предположению индукции найдется элементарное множество, скажем $\mathfrak{A}$, такое, что множество $\{c\} \times \mathfrak{E}_{n}$ является слоем $\mathfrak{A}$ и справедливы свойства $\left(b_{7 n}\right)$ и $\left(h_{5 n}\right)$. Заметим, что когда при фиксированном $c^{\prime} \in \mathscr{P}(\mathfrak{A})$ пара $\left(c^{\prime}, b\right)$ изменяется в пределах соответствуюшего слоя множества $\mathfrak{A}$, критическое значение $S(1)$ пробегает критический интервал $\varkappa_{n}\left(c^{\prime}\right)$, гладко зависящий от $\left(c^{\prime}, b\right)$ вследствие свойства $\left(b_{7 n}\right)$. Не ограничивая общности, можно считать, что и остальные критические интервалы гладко зависят от $\left(c^{\prime}, b\right)$, пока находимся в пределах $\mathfrak{A}$. Принимая во внимание это, а также свойства $\left(b_{7 n}\right)$ и $\left(b_{7(n-3)}\right)$, делаем вывод, что интервалы глубины $n+1$, создаваемые на шаге $n+1$, и интервалы множества $\mathfrak{p}_{n+1}$ обладают свойством гладкости в $\left(b_{7 n}\right)$. Соответственно новые $\lambda$-интервалы удовлетворяют требованию $\left(h_{5 n}\right)$. Отметим, что использование в этом месте свойства $\left(b_{7(n-3)}\right)$ оправдывается тем обстоятельством, что последовательность $\mathfrak{D}_{n}, n \in \mathbb{N} \cup\{0\}$, убывает. Действительно, тогда можно считать, что элементарные множества, построенные в доказательстве леммы 16 на предыдущих шагах, обладают соответствующим свойством вложенности.

В силу очевидных аналитических соображений из сказанного выводится лемма 16 для точек множества $\{c\} \times\left(\mathfrak{D}_{n+1}(c) \cap \mathfrak{E}_{n}\right)$, причем все элементарные множества $\mathfrak{A}^{\prime}$, при этом возникающие, имеют ту же проекцию, что и множество $\mathfrak{A}$.

Остается проверить свойства $\left(b_{7(n+1)}\right)$ и $\left(h_{5(n+1)}\right)$. Отметим, что первое из них уже фактически обсуждалось в отношении интервалов глубины $n+1$. Зафиксируем одно из множеств $\mathfrak{A}^{\prime}$. Выполняя на основании свойств $\left(b_{7 n}\right)$ и $\left(h_{5 n}\right)$ выбор точки $\widetilde{y}_{n+1}$, получаем интервал $\lambda_{n+1}$, гладко зависящий от параметров на элементарном множестве $\mathfrak{A}^{\prime \prime} \subset \mathfrak{A}^{\prime}$, в соответствии с $\left(h_{5(n+1)}\right)$. При этом, вообще говоря, проекция $\mathscr{P}\left(\mathfrak{A}^{\prime \prime}\right)$ может оказаться собственным подынтервалом $\mathscr{P}\left(\mathfrak{A}^{\prime}\right)$. Рассмотрим интервалы множества $\mathfrak{k}_{n+1} \backslash \mathfrak{k}_{n}$, расположенные внутри множества $\lambda_{n} \backslash \lambda_{n+1}$. Кроме интервалов множества $\mathfrak{n}_{n}$, сформированного к шагу $n+1$ (которые автоматически удовлетворяют требованию $\left(b_{7(n+1)}\right)$ в силу $\left(b_{7 n)}\right)$, внутри множества $\lambda_{n} \backslash \lambda_{n+1}$ расположены как интервалы, полученные в результате заполнений, так и интервалы, возникающие за счет взятия дополнительных композиций при выборе интервала $\lambda_{n+1}$. Последние, очевидно, обладают свойством $\left(b_{7(n+1)}\right)$, если эти композиции делать согласованно для всех $c^{\prime} \in \mathscr{P}\left(\mathfrak{A}^{\prime}\right)$. Если $\xi$ есть $\lambda$-интервал, заполняемый на шаге $n+1$, и $\xi$ отображается на $\delta_{l}, l<n-1$, под действием диффеоморфизма $f$, то по соображениям непрерьвности (свойство $\left.\left(h_{5 l}\right)\right)$ число $l$ одно и то же для любого $c^{\prime} \in \mathscr{P}\left(\mathfrak{A}^{\prime}\right)$. Учитывая свойства $\left(b_{7 l}\right)$ (для интервалов мно- 
жества $\left.\mathfrak{n}_{l}\right)$ и $\left(b_{7(l+1)}\right)$ (для интервалов множества $\left.\mathfrak{k}_{l}\right)$, делаем вывод, что интервалы множества $\mathfrak{k}_{n+1}$, получаемые в результате заполнения интервала $\xi$, обладают свойством $\left(b_{7(n+1)}\right)$. Новые $\lambda$-интервалы внутри $\xi$ и интервалы $\widehat{\xi}_{s}$ гладко зависят от параметров вследствие $\left(h_{5(l+1)}\right)$. Интервал $\widehat{\xi}_{p}$ совпадает с $\xi$ и, стало быть, тоже ведет себя регулярно по $c, b$ на множестве $\mathfrak{A}^{\prime}$. Для $\lambda$-интервалов, расположенных вне $\lambda_{n}$, рассуждения полностью аналогичны. Лемма доказана.

\section{§3. Доказательство теоремы 2}

3.1. Определение функций $a_{i}$. Заметим, что $d$ представляет собой минимальное значение $r_{a, d}(1)$ функции $r_{a, d}$ и что для любого $d$

$$
\partial_{a} r_{a, d}(x)>0, \quad \lim _{a \rightarrow \infty} r_{a, d}(x)=\infty, \quad x \neq 1 .
$$

Зафиксируем $d<1$ и будем, монотонно и непрерывно меняя параметр $a$ от $a_{0}$ до $\infty$, следить за поведением графика функции $r_{a, d}$. При этом встретим ряд примечательных значений параметра $a$, которые будут соответствовать бифуркациям между теми или иными типами поведения соответствующей динамической системы и определять искомые функции. Опишем эти значения.

Из того, что $r_{a, d}(1)=d<1, r_{a, d}(0)=d+a / 2>0$, и из соображений непрерывности и монотонности вытекает сушествование единственной неподвижной точки $p_{1}=p_{1}(a, d)$ отображения $r_{a, d}$ на интервале $(0,1)$, причем для любого $d$

$$
\lim _{a \rightarrow \infty} p_{1}(a, d)=1, \quad \partial r_{a, d}\left(p_{1}\right)<0, \quad \lim _{a \rightarrow \infty} \partial r_{a, d}\left(p_{1}\right)=-\infty
$$

Кроме того,

$$
\lim _{a \rightarrow a_{0}} p_{1}(a, d)=\max (d, 0), \quad \lim _{a \rightarrow a_{0}} \partial r_{a, d}\left(p_{1}\right)=-a_{0} .
$$

Функция $a_{1}$. Зафиксируем $d \in(-1 / 2,1)$ и определим функцию $a_{1}:(-1 / 2,1) \rightarrow \mathbb{R}$ следующим образом. Предположим, что

$$
\partial_{a} p_{1}>0, \quad \partial_{a}\left(\partial r_{a, d}\left(p_{1}(a, d)\right)\right)<0 .
$$

Тогда из соотношений (3.3) и (3.4) и неравенства $0 \leqslant a_{0}<1$ вытекает существование единственного значения $a_{1}=a_{1}(d) \in\left(a_{0}, \infty\right)$ такого, что $\partial r_{a_{1}, d}\left(p_{1}\right)=-1$. Функция $a_{1}$ описывает бифуркацию удвоения периода. При этом имеем

$$
r_{a_{1}, d}^{2}(1) \leqslant 1
$$

Действительно, при $\left|\partial r_{a, d}\left(p_{1}\right)\right|<1$ критическая точка $x=1$ должна принадлежать области притяжения точки $p_{1}$ (см. [1; с. 1028]), стало быть, $r_{a, d}^{2}(x)<x$ на интервале $\left(p_{1}, 1\right)$.

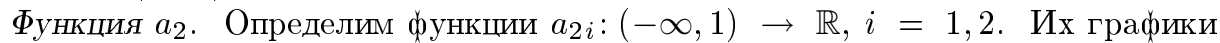
в плоскости $\{(a, d)\}$ соответствуют ситуации, когда вторая или третья итерация одной из критических точек попадает в отталкивающую неподвижную точку $p_{1}$. По определению для любого $d<1 a_{21}=a_{21}(d)\left(a_{22}=a_{22}(d)\right)$ представляет собой единственное число из интервала $\left(a_{0}, \infty\right)$ такое, что

$$
\begin{aligned}
r_{a, d}^{2}(1) & =\frac{1}{p_{1}} \\
\left(r_{a, d}(-1)\right. & \left.=\frac{1}{p_{1}}\right) .
\end{aligned}
$$


Это определение корректно, так как при изменении $a$ от $a_{0}$ до $\infty r_{a, d}(-1)$ строго возрастает (см. оценку (3.1)) от $\max (d,-d)$ до $+\infty$ и $r_{a, d}^{2}(1)=r_{a, d}(d)$ (соответственно $\left.1 / p_{1}\right)$ изменяется монотонно от $d$ до $+\infty$ (соответственно от $1 / d$ до 1$)$ в случае, когда $0 \leqslant d<1$, и от некоторого $r_{a_{0}, d}(d)$ до $+\infty($ соответственно от $+\infty$ до 1$)$, если $d<0$. Из соотношений (3.1), (3.5), (3.6) вытекает, что для любого $d \in(-1 / 2,1) a_{1}(d)<a_{21}(d)$. Так как при $d=-1 r_{a, d}^{2}(1)=r_{a, d}(-1)$, то имеем $a_{21}(-1)=a_{22}(-1)$. Кроме того, можно показать, что

$$
\lim _{d \rightarrow 1-0} a_{21}(d)=\lim _{d \rightarrow-\infty} a_{22}(d)=+\infty .
$$

Поэтому график функции $a_{2}:(-\infty, 1) \rightarrow \mathbb{R}$, определяемой равенством

$$
a_{2}(d)= \begin{cases}a_{21}(d), & d \in(-1,1) \\ a_{22}(d), & d \leqslant-1\end{cases}
$$

разбивает параметрическую плоскость на две односвязные области.

$\Phi$ ункция $a_{3}$. Для любого $d<1$ из соотношений $(3.1)$ и диффференцируемости $r_{a, d}$ следует наличие единственного числа $a^{\prime}=a^{\prime}(d)>a_{0}$ такого, что график функции $r_{a^{\prime}, d}$ касается диагонали в точке $(q, q), q=q(d) \in(1,+\infty)$. Это означает, что когда значение параметра $a$ изменяется вблизи $a^{\prime}$, возникают две неподвижные точки $p_{2}=p_{2}(a, d)$ и $p_{3}=p_{3}(a, d)$ отображения $r_{a, d}$ такие, что $1<p_{2}<p_{3}$, причем

$$
\partial_{a} p_{2}(a, d)<0, \quad \partial r_{a, d}\left(p_{2}\right)>1>\partial r_{a, d}\left(p_{3}\right)>0 .
$$

График функции $a_{3}$ представляет собой множество решений уравнения

$$
r_{a, d}^{2}(1)=p_{2}(a, d)
$$

Ясно, что равенство (3.10) влечет условие $d>0$. Действительно, в противном случае это равенство невозможно, ибо $r_{a, d}^{2}(1)=r_{a, d}(d) \geqslant r_{a, d}(0)>r_{a, d}(x)$ для любого $x>1$. Пусть $d \in(0,1)$. Возможны два случая: либо

$$
r_{a^{\prime}, d}^{2}(1) \leqslant q(d)
$$

либо, напротив, $r_{a^{\prime}, d}^{2}(1)>q(d)$. Сравнение производных $\partial_{a} r_{a, d}^{2}(1)$ и $\partial_{a} p_{2}(a, d)($ см. оценки (3.1) и (3.9)) говорит о том, что неравенство (3.11) эквивалентно существованию решения уравнения (3.10), причем решение единственно. Обозначим его через $a_{3}=a_{3}(d)>0$.

Лемма 17. Найдется $d^{\prime} \in(0,1)$ такое, что решением неравенства $(3.11)$ служит множество $\left[d^{\prime}, 1\right)$.

ДокАЗАТЕЛьство. Заметим, что решение уравнения

$$
r_{a^{\prime}, d}^{2}(1)=q(d)
$$

на интервале $(0,1)$ сушествует, единственно и приближенно равно 0.317183. Это легко установить, решая с помощью соответствуюшей программы эквивалентную систему соотношений

$$
r_{a^{\prime}, d}(d)=q, \quad \partial r_{a^{\prime}, d}(q)=1, \quad r_{a^{\prime}, d}(q)=q
$$


Обозначим решение через $d^{\prime}$. Остается выяснить знак выражения $r_{a^{\prime}, d}^{2}(1)-q(d)$ на интервале $\left(d^{\prime}, 1\right)$. Для этого зафиксируем $a=a^{\prime}\left(d^{\prime}\right)$ и будем менять с единичной скоростью параметр $d$ от $d^{\prime}$ до 1 . При этом очевидно, что для значений $d$, близких к $d^{\prime}$, выполнено $r_{a^{\prime}, d}^{2}(1)>p_{2}(a, d)$, так как при рассматриваемой деформации в начальный момент $d^{\prime}$ точки $p_{2}$ и $p_{3}$ движутся в разных направлениях с бесконечной скоростью. Заметим, что $\lim _{d \rightarrow 1-0} r_{a^{\prime}, d}^{2}(1)=1$ и $p_{2}(a, d) \geqslant p_{2}(a, 1)>1$ на интервале $\left(d^{\prime}, 1\right)$, поэтому соображения непрерывности влекут наличие решения $d_{0}$ уравнения $(3.10)$ на интервале $\left(d^{\prime}, 1\right)$. Так как $d_{0}$ есть решение неравенства $(3.11)$, то лемма доказана.

Таким образом, функция $a_{3}$ определена на промежутке $\left[d^{\prime}, 1\right)$. Заметим, что для любых $d<1$ и $a \geqslant a^{\prime}(d)$ имеем

$$
1 / p_{1}<p_{2}
$$

в силу возрастания $r_{a, b}$ на промежутке $[1,+\infty)$ и свойства $(7)$ функции $s_{a, b}$. Поэтому для любого $d \in\left(d^{\prime}, 1\right)$ вьполнено неравенство $a_{2}(d)<a_{3}(d)$. Это очевидно, если $a_{2}<a^{\prime}$, и вытекает из неравенства (3.12) и определения функции $a_{22}$ в противном случае.

Проверим неравенства (3.4). Так как $0<p_{1}<1$ и $p_{1}=r_{a, d}\left(p_{1}\right)>r_{a, d}(1)=d$, то имеем

$$
\partial_{a} p_{1}(a, d)=\left.\frac{1-p}{2 a(1-p)+4 p(p-d)+2\left(1+p^{2}\right)}\right|_{p=p_{1}(a, d)}>0
$$

Обозначив $\varrho(x)=\frac{1}{2} \frac{(1-x)^{2}}{1+x^{2}}$, запишем

$$
\partial_{a}\left(\partial r_{a, d}\left(p_{1}(a, d)\right)\right)=\varrho^{\prime}(p)+\left.a \varrho^{\prime \prime}(p) \frac{\varrho(p)}{1-a \varrho^{\prime}(p)}\right|_{p=p_{1}(a, d)} .
$$

С учетом последней формулы и того, что $\varrho^{\prime}\left(p_{1}\right)<0$, второе из неравенств $(3.4)$ будет вытекать из соотношения $\varrho^{\prime \prime} \varrho<\left(\varrho^{\prime}\right)^{2}$ на интервале $(0,1)$, которое проверяется непосредственно.

3.2. Доказательство теоремы 2. Утверждение (iii) теоремы доказано в работе [1].

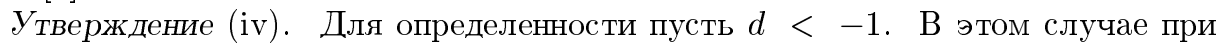
$a>a_{2}(d)$ имеем $r_{a, d}(1)<-1$ и $r_{a, d}(-1)>1 / p_{1}$. Это означает, что на отрезке $J=\left[p_{1}, p_{1}^{-1}\right]$ функция $r_{a, d}^{2}$ имеет две точки максимума $x_{1}, x_{2}$ и точку локального минимума $x=1$, причем $x_{1}<1<x_{2}$ и $r_{a, d}^{2}\left(\left[p_{1}, x_{1}\right]\right)=r_{a, d}^{2}\left(\left[x_{2}, p_{1}^{-1}\right]\right)=$ $\left[p_{1}, r_{a, d}(-1)\right] \supset J$. Стало быть, отображение $r_{a, d}^{2}$ обладает подковой (см. [6]), что и требовалось доказать. Случай $d \in[-1,1)$ рассматривается аналогично.

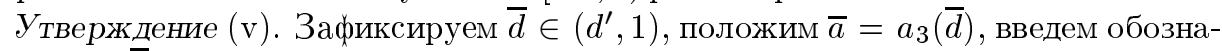
чение $d(a, \bar{d})$ для выражения

$$
\bar{d}^{-1}-\frac{a}{2} \frac{(1-\bar{d})^{2}}{1+\bar{d}^{2}}
$$

и рассмотрим луч $\mathfrak{K}_{\bar{d}}=\{(a, d) \in \mathbb{R}: a \leqslant \bar{a}, d=d(a, \bar{d})\}$. Для любой пары $(a, d) \in \mathfrak{K}_{\bar{d}}$ отрезок $J_{\bar{d}}=\left[\bar{d}, \bar{d}^{-1}\right]$ инвариантен относительно $r_{a, d}$, причем $\bar{d}^{-1}-$ неподвижная точка $r_{a, d}$ (отталкивающая при $a$, близких к $\bar{a}$ ), $\bar{d}$ - ее прообраз (все 
сказанное вытекает из равенств $(1.1),(1.2)$ и свойства $(6)$ функции $\left.s_{a, b}\right)$. Непосредственно проверяется, что для любого $0<a \leqslant \bar{a}$ отображение $r_{a, d(a, \bar{d})}: J_{\bar{d}} \rightarrow J_{\bar{d}}$ аффиннно сопряжено с отображением $w_{a}:\left[0, \bar{d}^{-1}-\bar{d}\right] \rightarrow\left[0, \bar{d}^{-1}-\bar{d}\right]$, определяемым по формуле

$$
w_{a}(x)=a\left(\frac{1}{2} \frac{(1-\bar{d})^{2}}{1+\bar{d}^{2}}-\frac{1}{2} \frac{\left(1-\bar{d}^{-1}+x\right)^{2}}{1+\left(\bar{d}^{-1}-x\right)^{2}}\right)
$$

с помощью преобразования $x \mapsto \bar{d}^{-1}-x, x \in\left[0, \bar{d}^{-1}-\bar{d}\right]$. Так как свойство отрицательности шварциана выживает при аффинной замене переменной, то в данной ситуации примени́м результат работы [12; теорема 2.2] и сушествует множество положительной лебеговой меры $\mathfrak{U}_{\bar{d}} \in \mathbb{R}$ такое, что для любого $a \in \mathfrak{U}_{\bar{d}}$ отображение $r_{a, d(a, \bar{d})}$ имеет к.а.н.и. меру, причем число $\bar{a}$ является левосторонней точкой плотности множества $\mathfrak{U}_{\bar{d}}$. Тогда

$$
\mathfrak{S}_{2}=\bigcup_{\bar{d} \in\left(d^{\prime}, 1\right)}\left\{(a, d) \in \mathbb{R}^{2}: a \in \mathfrak{U}_{\bar{d}}, d=d(a, \bar{d})\right\}
$$

(ср. с соответствующим местом в доказательстве теоремы 1).

3.3. Выражения для функций $a_{i}$. В заключительной части статьи будут проделаны некоторые полезные вычисления.

$\Phi$ ункция $a_{1}$. Воспроизведем соответствуюшие формулы из [1]. Из определения $a_{1}$ вытекает, что равенство $a=a_{1}(d)$ эквивалентно системе соотношений

$$
r_{a, d}(p)=p, \quad \partial r_{a, d}(p)=-1, \quad p>0
$$

Исключая $p$, находим

$$
d=p-a \varrho(p), \quad a>1
$$

где

$$
p=\left(\frac{-a-2+\sqrt{a(8+a)}}{2}\right)^{1 / 2}
$$

Функция $a_{2}$. Нетрудно установить путем нескольких преобразований, что условие $a=a_{22}(d)$ эквивалентно системе соотношений

$$
a=\frac{2}{p} \frac{1-p}{1+p}\left(1+p^{2}\right), \quad d=\frac{1}{p}-\frac{2}{p} \frac{1-p}{1+p}\left(1+p^{2}\right), \quad 0<p<1
$$

причем $p=p_{1}(a, d)$. В соответствии с равенством (3.8) остается выяснить, при каких $p d(p) \leqslant-1$.

ЛЕмма 18. Функция $p_{1}(d)=p_{1}\left(a_{22}(d), d\right)$ строго возрастает от 0 до 1 на интервале $(-\infty, 1)$. 
ДокАЗАТЕльство. Предположим противное, тогда либо $p_{1}(d)$ убывает, либо существуют $d_{1}$ и $d_{2}, d_{1}<d_{2}$, такие, что $p_{1}\left(d_{1}\right)=p_{1}\left(d_{2}\right)=p$. Первое невозможно, ибо $p_{1}(1-0)=1$. Второе ведет к противоречию. Пусть $r_{i}=\left.r_{a_{22}\left(d_{i}\right), d_{i}}\right|_{[-1,1]}$, $i=1,2$. Тогда функции $r_{i}$ удовлетворяют условиям

$r_{1}(1)<r_{2}(1), \quad r_{1}(-1)=r_{2}(-1)=p^{-1}, \quad r_{1}(p)=r_{2}(p)=p, \quad r_{1}^{\prime}=C r_{2}^{\prime}, \quad C>0$

которые несовместимы.

Решая уравнение $d(p)=-1$, находим единственное $p^{\prime} \approx 0.258056$ такое, что формулы (3.15) при $0<p \leqslant p^{\prime}$ представляют собой параметрическое представление сужения $\left.a_{2}\right|_{(-\infty,-1]}$.

Функция $a_{3}$. Заметим, что равенство $a=a_{3}(d)$ эквивалентно тому, что $r_{a, d}(d)=$ $d^{-1}, d \in\left(d^{\prime}, 1\right)$ (см. свойство $(7)$ функции $\left.s_{a, b}\right)$. Решая это уравнение, находим

$$
a_{3}(d)=\frac{2}{d} \frac{1+d}{1-d}\left(1+d^{2}\right), \quad d \in\left(d^{\prime}, 1\right) .
$$

\section{Список литературы}

1. O'Cairbre F., O'Farrell A. G., O'Reilly A. Bistability, bifurcation and chaos in a laser system // Internat. J. Bifur. Chaos Appl. Sci. Engrg. 1995. V. 5. № 4. P. 1021-1031.

2. Jakobson M. Absolutely continuous invariant measures for one-parameter families of one-dimensional maps // Comm. Math. Phys. 1981. V. 81. P. 39-88.

3. Costa M.J. Chaotic behaviour of one-dimensional saddle-node horseshoes // Preprint CMA, 2001.

4. Homburg A.J., Young T. Jakobson's theorem near saddle-node bifurcations // Preprint, 2001.

5. Aaronson J. An introduction to infinite ergodic theory. Providence: Amer. Math. Soc., 1997. (Math. Surveys Monogr. V. 50.)

6. Katok A., Hasselblatt B. Introduction to the modern theory of dynamical systems. Cambridge: Cambridge Univ. Press, 1995.

7. Benedicks M., Carleson L. The dynamics of the Hénon map // Ann. of Math. (2). 1991. V. 133. № 1. P. 73-169.

8. Jakobson M., Światek G. Metric properties of non-renormalizable $S$-unimodal maps. I. Induced expansion and invariant measures // Ergodic Theory Dynam. Systems. 1994. V. 14. № 4. P. 721-755.

9. Jakobson $M$. Uniformly scaled Markov partitions for unimodal maps // J. Math. Sci. (New York). 1999. V. 95. № 5. P. 2583-2608.

10. Ахалая ШІ. И., Степин А. М. Об инвариантных мерах несжимающих отображений // Сообщения АН Грузии. 1980. Т. 100. № 3. С. 549-552.

11. Young L.-S. Recurrence times and rates of mixing // Preprint, 1997.

12. Якобсон M. В. Эргодическая теория одномерных отображений // Итоги науки и техники. Соврем. пробл. матем. Фундам. напр. Т. 2. М.: ВИНИТИ, 1985. С. 204-226. 